\title{
Images of Rational Maps of Projective Spaces.
}

\author{
BINGLIN LI
}

July 10, 2018

\begin{abstract}
Consider a rational map from a projective space to a product of projective spaces, induced by a collection of linear projections. Motivated by the the theory of limit linear series and Abel-Jacobi maps, we study the basic properties of the closure of the image of the rational map using a combination of techniques of moduli functors and initial degenerations. We first give a formula of multi-degree in terms of the dimensions of intersections of linear subspaces and then prove that it is Cohen-Macaulay. Finally, we compute its Hilbert polynomials.
\end{abstract}

Keywords: projective spaces, moduli functors, multi-degrees, Chow rings, initial ideals, Hilbert polynomials. 


\section{INTRODUCTION}

\section{Main Result}

Let $V$ be a vector space over an algebraically closed field $k$, and consider the following rational map:

$$
f: \mathbb{P}(V) \rightarrow \Pi_{i=1}^{n} \mathbb{P}\left(V / V_{i}\right),
$$

which is induced by the choice of linear subspaces $V_{i} \subset V$. Let $X\left(V_{1}, \cdots, V_{n}\right)$ be the closure of the image of $f$. The purpose of this paper is to study the basic properties of $X\left(V_{1}, \cdots, V_{n}\right)$, using techniques of moduli functors and initial degenerations.

Fix a choice of $n$ linear subspaces $\left\{V_{i}\right\}_{i=1}^{n}$ with $\bigcap_{i=1}^{n} V_{i}=\{0\}$, and let $d_{I}$ be the dimension of $\cap_{i \in I} V_{i}$ and define $M(p)$ to be the set:

$$
\left\{\left(m_{1}, \cdots, m_{n}\right) \in \mathbb{Z}_{\geq 0}^{n}: r+1-\sum_{i \in I} m_{i}>d_{I} \forall I \subset[n], \sum_{i=1}^{n} m_{i}=p\right\}
$$

In this paper, we have:

Theorem 1.1. - Set $p=\max \{h ; M(h) \neq \emptyset\}$. The dimension of $X\left(V_{1}, \cdots, V_{n}\right)$ is $p$. Its multi-degree function takes value one at the integer vectors in $M(p)$ and zero otherwise. The Hilbert Polynomial of $X\left(V_{1}, \cdots, V_{n}\right)$ is

$$
\sum_{S \subset M(p)}(-1)^{|S|-1} \Pi_{i=1}^{n}\left(\begin{array}{c}
u_{i}+\ell_{S, i} \\
\ell_{S, i}
\end{array}\right)
$$

where $u_{i}$ 's are the variables and $\ell_{S, i}$ is the smallest $i$-th component of all elements of $S$. Moreover, $X\left(V_{1}, \cdots, V_{n}\right)$ is Cohen-Macaulay.

\section{Background and Motivation}

Questions concerning some basic geometric properties of $X\left(V_{1}, \cdots, V_{n}\right)$ naturally arise in many settings, some of which are detailed below.

First, in [5] by Esteves and Osserman, the closures of rational maps of the following form

$$
\mathbb{P}(V) \rightarrow \mathbb{P}\left(V / V_{1}\right) \times \mathbb{P}\left(V / V_{2}\right)
$$

arise as irreducible components of the closed subscheme of the fiber of the Abel map associated to a limit linear series. Moreover, the union of those irreducible components forms a flat degeneration of a projective space. In this work, they work with singular curves of compact type with two components, so they only need to study the closure of the image in a product of two projective spaces, where $V_{1} \cap V_{2}=\{0\}$. 
Secondly, in computer vision [6] by Aholt, Sturmfels and Thomas, an important theme is to study the geometry of the closure of the rational map:

$$
\mathbb{P}^{3} \rightarrow\left(\mathbb{P}^{2}\right)^{n}
$$

In their case, all the $V_{i}$ 's are one dimensional and have pairwise trivial intersection.

Moreover, in the theory of Mustafin varieties in [7] and [4], flat degenerations of $\mathbb{P}^{r}$ are carefully studied, and the spaces $X\left(V_{1}, \cdots, V_{n}\right)$ arise as irreducible components of the Mustafin degenerations.

Finally, $X\left(V_{1}, \cdots, V_{n}\right)$ manifest themselves in algebraic statistics in [3] by Morton .

Despite the ubiquity of the spaces $X\left(V_{1}, \cdots, V_{n}\right)$, a systematic study of its the geometry (multidegree, singularity type, initial degeneration, Hilbert Polynomial etc.) has not be conducted in full generality. Such generality is needed. The author's original motivation is to generalize the work of Esteves and Osserman to arbitrary curves of compact type. In this case, one cannot just focus on the map to a product of two projective spaces, and also the vector subspaces $V_{i}$ 's do not mutually just have trivial intersections anymore, but rather the only restriction we may impose is that $\bigcap_{i=1}^{n} V_{i}=\{0\}$. We don't put restrictions on the dimension of $V$ and $V_{i}$ 's. Moreover, we hope that our generality can lead to better understanding of irreducible components of Mustafin Degenerations.

\section{Structure of the Paper}

In Section II, we will describe two closed subschemes $\mathscr{Z}_{1}$ and $\mathscr{Z}_{2}$ of $\Pi_{i=1}^{n} \mathbb{P}\left(V / V_{i}\right)$, and prove that they are equal and that they agree with $X\left(V_{1}, \cdots, V_{n}\right)$ set-theoretically.

In Section III we will compute the multi-degree of $\mathscr{Z}_{1}$. It seems to be an elementary linear algebra argument, but the proof involves moduli-theoretic techniques to exhibit the existence of the system of linear subspaces satisfying the desired property and such technique is also used to compute the deformation.

In the Section IV we will compute the initial degeneration of $\mathscr{Z}_{2}$ under a prescribed term order. The slogan is that multi-degrees determine the initial degenerations of $X\left(V_{1}, \cdots, V_{n}\right)$. We also prove that $\mathscr{Z}_{1}, \mathscr{Z}_{2}$ and $X\left(V_{1}, \cdots, V_{n}\right)$ are isomorphic as schemes, and finally conclude that $X\left(V_{1}, \cdots, V_{n}\right)$ is Cohen-Macaulay.

Section V will be devoted to computing the multi-variable Hilbert polynomials. Our computation of the Hilbert polynomial is not by counting monomials using the information of the initial ideal obtained in Section III, but rather by directly computing the Hilbert polynomial of its initial degeneration, which is a union of product of projective spaces whose intersections are also products of projective spaces, which is easier and more intuitive to deal with.

\section{Acknowledgements}

The author would like to thank his adviser Brian Osserman for his detailed and patient guidance. The author also wants to thank Professor Bernd Sturmfels for helpful discussions relating this work 
with [7] and [3], and also Professor Allen Knutson for introducing the author [1] which reduces problem of "Cohen-Macaulay"ness to the proof of "multiplicity free".

Also special thanks to Naizhen Zhang and Christopher Westenberger for encouraging my studies in algebraic geometry and Federico Castillo for explaining to me some foundational material in combinatorial commutative algebra and useful discussions. The author also wants to thank Michael Gröchenig for carefully proofreading this paper.

\section{DESCRIPTIONS OF $X\left(V_{1}, \cdots, V_{n}\right)$}

\subsection{Set-theoretic Description of $X\left(V_{1}, \cdots, V_{n}\right)$}

In this section, we first give a set theoretic description of $X\left(V_{1}, \cdots, V_{n}\right)$. A scheme theoretic description of $X\left(V_{1}, \cdots, V_{n}\right)$ will be given in Section 2.3. Observing that $k$-valued points of $\Pi_{i=1}^{n} \mathbb{P}\left(V / V_{i}\right)$ correspond to $n$ - tuples $\left(W_{1}, \cdots, W_{n}\right)$, where each $W_{i}$ is a linear subspace of $V$ which contains $V_{i}$ as codimension one linear subspace, we have the following theorem.

Proposition 2.1. - The closure of the image of the rational map $\mathbb{P}(V) \rightarrow \Pi_{i=1}^{n} \mathbb{P}\left(V / V_{i}\right)$ are settheoretically in bijection with n-tuples $\left(W_{1}, \cdots, W_{n}\right)$ where $W_{i}$ contains $V_{i}$ as a codimension one subspace and for any $I \subset[n]$, we have $\bigcap_{I} W_{i} \supsetneqq \bigcap_{I} V_{i}$.

Before proving the statement, let's first start with an example:

Example 2.1. - Let $V$ be a five-dimensional linear subspace over a field $k$ with basis $\left\{e_{i}\right\}_{i=1}^{5}$. Let $V_{1}=\operatorname{span}\left\{e_{1}, e_{2}\right\}, V_{2}=\operatorname{span}\left\{e_{1}, e_{3}\right\}$, and $V_{3}=\operatorname{span}\left\{e_{5}\right\}$. Let $W_{1}=V_{1} \oplus \operatorname{span}\left\{e_{3}\right\}, W_{2}=V_{2} \oplus$ $\operatorname{span}\left\{e_{4}\right\}$ and $W_{3}=V_{3} \oplus \operatorname{span}\left\{e_{1}\right\}$.

We have $e_{1} \in W_{1} \cap W_{2} \cap W_{3}$, but $e_{1} \in\left(V_{1} \cap V_{2}\right) \backslash V_{3}$, which means $\left(W_{1}, W_{2}, W_{3}\right)$ is not in the image of $f$. We also have $e_{3} \in W_{1} \cap W_{2}$, and $e_{3} \in V_{2} \backslash V_{1}$. Consider the following one-parameter family: $\mathscr{W}_{t}=\left(\operatorname{Span}\left(V_{1},\left(e_{3}+t e_{4}\right)\right), W_{2}, \operatorname{Span}\left(V_{3},\left(e_{1}+t e_{3}+t^{2} e_{4}\right)\right)\right.$. When $t=0$, it is the point $\left(W_{1}, W_{2}, W_{3}\right)$, when $t \neq 0, \mathscr{W}_{t} \in \operatorname{Im} f$ except for possibly finite many values of $t$. In this example, $\left(W_{1}, W_{2}, W_{3}\right)$ is not in the image of $f$, but is in the closure of the image of $f$

\section{Proof of Proposition 2.1.}

Note that $k$-valued points in the image of the rational map $\mathbb{P}(V) \rightarrow \Pi_{i=1}^{n} \mathbb{P}\left(V / V_{i}\right)$ correspond to $\left(\operatorname{Span}\left(V_{1}, v\right), \cdots, \operatorname{Span}\left(V_{n}, v\right)\right)$ where $v \in V \backslash\left(\cup_{i=1}^{n} V_{i}\right)$, thus one containment follows. For the other containment, given $C_{0}=\left(W_{1}, \cdots, W_{n}\right)$ satisfying $\cap_{i \in I} W_{i} \supsetneqq \cap_{i \in I} V_{i}$, for all $I \subset[n]$, we will construct a one-parameter family $C_{t}$, such that when $t \neq 0$, we have $C_{t}=\left(\operatorname{Span}\left(V_{1}, v_{t}\right), \cdots, \operatorname{Span}\left(V_{n}, v_{t}\right)\right)$ with $v_{t} \in V \backslash\left(\cup_{i=1}^{n} V_{i}\right)$ except for possibly finite many values of $t$, and $C_{t}=C_{0}$ when $t=0$. 
Let $I_{0}=[n]=\{1,2 \cdots, n\}$, then take a $w_{0} \in \cap_{i \in[n]} W_{i} \backslash \cap_{i=1}^{n} V_{i}$. Let $I_{1}=\left\{i \mid w_{0} \in V_{i}\right\}$. Then take a $w_{1} \in \bigcap_{I_{1}} W_{i} \backslash \bigcap_{I_{1}} V_{i}$, and let $I_{2}=\left\{i \mid w_{1} \in V_{i}, i \in I_{1}\right\}$, and take $w_{2} \in \bigcap_{i \in I_{2}} W_{i} \backslash\left(\bigcap_{i \in I_{2}} V_{i}\right)$. Repeating this process, i.e. choose a $w_{j} \in \bigcap_{I_{j}} W_{i} \backslash \bigcap_{I_{j}} V_{i}$, and let $I_{j+1}=\left\{i \in I_{j} \mid w_{j} \in V_{i}\right\}$ so that we have

$$
I_{1} \supsetneqq I_{2} \cdots \supsetneqq I_{j} \cdots
$$

Each $I_{k}$ is finite, thus for some $m$ we have $I_{m+1}=\emptyset$. For $i \in I_{j} \backslash I_{j+1}$, we can express $W_{i}=\operatorname{Span}\left(V_{i}, w_{j}\right)$ (note that $i$ and $j$ are not necessarily the same).

Now consider the following set parameterized by $t \in k=\bar{k}$ :

It suffices to consider the one parameter family component wise: for component $i$, we can find $j$, such that $i \in I_{j} \backslash I_{j+1}$. Let $\left(W_{i}\right)_{t}=\operatorname{Span}\left(V_{i}, \sum_{j \leq \ell \leq m} t^{\ell-j} w_{\ell}\right)$.

When $t=0$, we have $C_{0}=\left(W_{1}, \cdots W_{n}\right)$ the point we start with. When $t \neq 0$, we have $\left(W_{i}\right)_{t}=$ $\operatorname{Span}\left(V_{i}, \sum_{j \leq \ell \leq m} t^{\ell-j_{w_{\ell}}}\right)=\operatorname{Span}\left(V_{i}, \sum_{0 \leq \ell \leq m} t^{\ell} w_{\ell}\right)$, thus $\left(\left(W_{1}\right)_{t}, \cdots,\left(W_{n}\right)_{t}\right)$ is in the image of $f$ except for possibly finitely many values of $t$. Then the proposition follows.

\subsection{Description II}

Given coordinate systems for all $V$ and $V / V_{i}$ 's, let $A_{i}$ be the matrix representation of the linear map $V \rightarrow V / V_{i}$, and $q_{i}$ be $\left(x_{i, 1}, \cdots, x_{i, r+1-d_{i}}\right)^{T}$. Set $I=\left\{\delta_{1}, \cdots, \delta_{|I|}\right\}$ to be a subset of $[n]$, and consider the following matrix associated to $I \subset[n]$,

$$
B_{I}=\left[\begin{array}{ccccc}
A_{\delta_{1}} & q_{\delta_{1}} & 0 & \ldots & 0 \\
A_{\delta_{2}} & 0 & q_{\delta_{2}} & \vdots & 0 \\
\vdots & \vdots & & & \\
A_{\delta_{|I|}} & 0 & \ldots & \ldots & q_{\delta_{|I|}}
\end{array}\right]
$$

Denote the ideal generated by all of the $r+1-d_{I}+|I|$-minors of $B_{I}$ as $\mathfrak{I}_{B_{I}}$, and denote the ideal generated by $\left\{\mathfrak{I}_{B_{I}} \mid I \subset[n]\right\}$ as $I_{f}$. Description II of $X\left(V_{1}, \cdots, V_{n}\right)$ is the closed subscheme of $\Pi_{i=1}^{n} \mathbb{P}\left(V / V_{i}\right)$ cut by $I_{f}$.

Definition 2.2. - Let I be a finite index set and $\left\{V_{i}\right\}_{i \in I}$ be a system of linear subspaces of $V$, and $V \supset W_{i} \supsetneqq V_{i}$ with $\operatorname{dim} W_{i}-\operatorname{dim} V_{i}=1, \forall i \in I$. Then define $g_{I}$ to be the following map:

$$
g_{I}: \quad V \bigoplus\left(\oplus_{i \in I} W_{i} / V_{i}\right) \rightarrow \oplus_{i \in I}\left(V / V_{i}\right)
$$

induced by the following data:

(1) $V \rightarrow V / V_{i}$ the natural quotient map.

(2) $g_{i, i}: W_{i} \rightarrow V / V_{i}$ is the natural composed map $W_{i} \hookrightarrow V \rightarrow V / V_{i}$, and $g_{i, j}: W_{i} \rightarrow V / V_{j}$ is the zero map for $i \neq j$. 
Given any $W_{i} \subset V$ which contains $V_{i}$ as a codimension one subspace, $W_{i} / V_{i}$ corresponds to a rational point of $\mathbb{P}\left(V / V_{i}\right)$ through the natural map $W_{i} / V_{i} \rightarrow V / V_{i}$. Conversely, given a rational point of $\mathbb{P}\left(V / V_{i}\right)$, which corresponds to a one-dimensional subspace $\ell$ of $V / V_{i}$, take the preimage of $\ell$ under the natural quotient map $V \rightarrow V / V_{i}$, then one get a linear subspace $W_{i}$ of $V$ containing $V_{i}$ as a codimension one subspace. Given a homogeneous coordinate of $\mathbb{P}\left(V / V_{i}\right)$, which is equivalent to giving a basis of $V / V_{i}$, the matrix representation of $s_{i}: W_{i} / V_{i} \rightarrow V / V_{i}$ is the same as the homogeneous coordinate of the point corresponding to $W_{i}$ up to a scalar. One can see that the matrix representation of $g_{I}$ is the same as $B_{I}$.

One can immediately get that Description II agrees with $X\left(V_{1}, \cdots, V_{2}\right)$ at least set-theoretically:

Proposition 2.3. - Let $V$ be a vector space over a field $K$ with dimension $(r+1)$. Let $I$ be a finite index set and $\left\{V_{i}\right\}_{i \in I}$ be a system of linear subspaces of $V$, and $V \supset W_{i} \supsetneqq V_{i}$ with $\operatorname{dim} W_{i}-\operatorname{dim} V_{i}=$ $1, \forall i \in I$. Then $\cap_{i \in I} W_{i} \supsetneqq \cap_{i \in I} V_{i}$ if and only if the following naturally induced map:

$$
g_{I}: \quad V \bigoplus\left(\oplus_{i \in I} W_{i} / V_{i}\right) \rightarrow \oplus_{i \in I}\left(V / V_{i}\right)
$$

has $\bigwedge^{\left(r+1-\operatorname{dim}\left(\cap_{i \in I} V_{i}\right)+|I|\right)} g_{I}=0$, where $g_{I}$ is defined in Definition 2.2

\section{Proof}

The condition $\bigwedge^{\left(r+1-\operatorname{dim}\left(\cap_{i \in I} V_{i}\right)+|I|\right)} g_{I}=0$ is equivalent to the condition that $g_{I}$ has nontrivial kernel. The kernel of $g_{I}$ is $\left(\cap_{i \in I} W_{i}\right) /\left(\cap_{i \in I} V_{i}\right)$. The kernel is nontrivial if and only if $\left(\cap_{i \in I} W_{i}\right) /\left(\cap_{i \in I} V_{i}\right) \neq 0$, which is equivalent to the condition that $\cap_{i \in I} V_{i} \supsetneqq \cap_{i \in I} W_{i}$.

\subsection{Equivalence of Two Descriptions}

In this section I will prove that not just set-level, but also as schemes, the two descriptions in subsection 2.1 and 2.2 are the same. What we have done essentially in Proposition 2.3 is compare $k$-valued points of the two descriptions. In this section, in order to compare the scheme structures of the two descriptions, we are going to compare their functor of points.

Before giving the definition of $\mathfrak{F}_{1}$ and $\mathfrak{F}_{2}$, we first define a moduli functor $\mathfrak{F}$, which contains $\mathfrak{F}_{1}$ and $\mathfrak{F}_{2}$ as closed sub-functors.

Definition 2.4. - Define $\mathfrak{F}$ to be a functor :

$$
(\mathbf{S c h} / \mathbf{k})^{\text {op }} \rightarrow \text { Sets }
$$

For any scheme $S / k$, we associate the set of tuples sub-vector bundles $\left(\mathscr{W}_{1}, \ldots, \mathscr{W}_{n}\right)$ of $\mathscr{V}_{S}:=V \otimes \mathscr{O}_{S}$ with $\operatorname{Rank}\left(\mathscr{W}_{i}\right)=\operatorname{dim} V_{i}+1$, for any $i \in[n]$. And for each $i \in[n]$, the sub-bundle $\mathscr{W}_{i}$ contains $V_{i} \otimes \mathscr{O}_{S}$ as a sub-bundle.

Note that the moduli functor $\mathfrak{F}$ represents nothing else than $\Pi_{i=1}^{n} \mathbb{P}\left(V / V_{i}\right)$. The following two functors $\mathfrak{F}_{1}$ and $\mathfrak{F}_{2}$ represent closed subschemes of $\Pi_{i=1}^{n} \mathbb{P}\left(V / V_{i}\right)$. 
Definition 2.5. - Define $\mathfrak{F}_{1}$ to be a functor :

$$
(\mathbf{S c h} / \mathbf{k})^{\text {op }} \rightarrow \text { Sets. }
$$

Given a scheme $S / k$, we associate the set of tuples sub-vector bundles $\left(\mathscr{W}_{1}, \ldots, \mathscr{W}_{n}\right)$ of $\mathscr{V}_{S}:=V \otimes \mathscr{O}_{S}$ with $\operatorname{Rank}\left(\mathscr{W}_{i}\right)=\operatorname{dim} V_{i}+1$ for any $i \in[n]$ such that:

(i) for each $i \in[n]$, the sub-bundle $\mathscr{W}_{i}$ also contains $V_{i} \otimes \mathscr{O}_{S}$ as a sub-bundle

(ii) for any $I \subset[n]$ and the following bundle map:

$$
f_{I}: \quad V /\left(\cap_{i \in I} V_{i}\right) \otimes \mathscr{O}_{S} \rightarrow \oplus_{i \in I} \mathscr{V} / \mathscr{W}_{i}
$$

we have

$$
\bigwedge^{\left(r+1-d_{I}\right)} f_{I}=0
$$

In order the define the second functor, we need the following lemma:

Lemma 2.6. - Let $V$ be a vector space over $k$ and $V^{\prime} \subset V$ is a sub vector space of $V$. For a scheme $S / k$, consider the following exact sequence:

$$
0 \rightarrow V^{\prime} \otimes \mathscr{O}_{S} \stackrel{i_{1}}{\longrightarrow} \mathscr{W} \stackrel{i_{2}}{\longrightarrow} V \otimes \mathscr{O}_{S}
$$

where $\mathscr{W}$ is a sub-bundle of $V \otimes \mathscr{O}_{S}$ with $\operatorname{Rank}(\mathscr{W})=\operatorname{dim} V^{\prime}+1$, and all of $i_{1}, i_{2}$ and $i_{2} \circ i_{1}$ have locally free cokernels. Denote $\mathscr{L}$ to be the cokernel of $i_{1}$, which is a line bundle. Then we have

(i) the induced morphism $0 \rightarrow \mathscr{W} \rightarrow\left(V / V^{\prime}\right) \otimes \mathscr{O}_{S}$ factors through $\mathscr{L} \rightarrow\left(V / V^{\prime}\right) \otimes \mathscr{O}_{S}$

(ii) this morphism $\mathscr{L} \rightarrow\left(V / V^{\prime}\right) \otimes \mathscr{O}_{S}$ is a sub-bundle morphism, i.e., has locally free cokernel

\section{Proof:}

For (i), consider the sequence $0 \rightarrow V^{\prime} \otimes \mathscr{O}_{S} \rightarrow \mathscr{W} \rightarrow V \otimes \mathscr{O}_{S} \rightarrow\left(V / V^{\prime}\right) \otimes \mathscr{O}_{S}$, then we get $0 \rightarrow V^{\prime} \otimes$ $\mathscr{O}_{S} \rightarrow \mathscr{W} \rightarrow\left(V / V^{\prime}\right) \otimes \mathscr{O}_{S}$ in which the morphism from $V \otimes \mathscr{O}_{S}$ to $\left(V / V^{\prime}\right) \otimes \mathscr{O}_{S}$ is zero. Therefore by the universal property of cokernel, we naturally get the following commutative diagram:

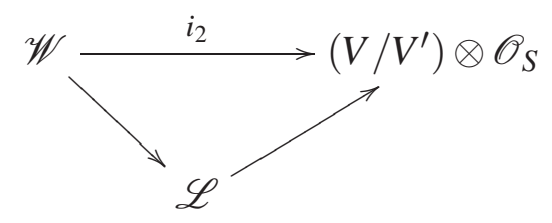

For (ii), it suffices to check locally at a point $p \in S / k$. The induced morphism $\mathscr{L} \rightarrow\left(V / V^{\prime}\right) \otimes \mathscr{O}_{S}$ is essentially the morphism $\mathscr{W} /\left(V^{\prime} \otimes \mathscr{O}_{S}\right) \rightarrow\left(V \otimes \mathscr{O}_{S}\right) /\left(V^{\prime} \otimes \mathscr{O}_{S}\right)$. Locally at $p$, we have

$$
0 \rightarrow\left(V^{\prime} \otimes \mathscr{O}_{S}\right)_{p} \underset{i_{1 p}^{\prime}}{\stackrel{i_{1 p}}{\rightleftarrows}} \mathscr{W}_{p} \underset{i_{2 p}^{\prime}}{\stackrel{i_{2 p}}{\rightleftarrows}}\left(V \otimes \mathscr{O}_{S}\right)_{p}
$$


such that $i_{1 p}^{\prime} \circ i_{1 p}=\mathrm{id}, i_{2 p}^{\prime} \circ i_{2 p}=\mathrm{id}$ and $\left(i_{2 p}^{\prime} \circ i_{1 p}^{\prime}\right) \circ\left(i_{2 p} \circ i_{1 p}\right)=\mathrm{id}$, because $i_{1}, i_{2}$ and $i_{2} \circ i_{1}$ have locally free cokernels by our assumption. Therefore,

$$
\left(\left(V \otimes \mathscr{O}_{S}\right)_{p} /\left(V^{\prime} \otimes \mathscr{O}_{S}\right)_{p}\right) /\left(\mathscr{W}_{p} /\left(V^{\prime} \otimes \mathscr{O}_{S}\right)_{p}\right) \cong\left(\left(V \otimes \mathscr{O}_{S}\right)_{p} / \mathscr{W}_{p}\right)
$$

Note that $\left(\left(V \otimes \mathscr{O}_{S}\right)_{p} / \mathscr{W}_{p}\right)$ is a free module over the local ring $\left(\mathscr{O}_{S}\right)_{p}$, thus our lemma follows.

The following functor $\mathfrak{F}_{2}$ is the functor of points for Description II.

Definition 2.7. - Let $\mathfrak{F}_{2}$ be a functor:

$$
(\mathbf{S c h} / \mathbf{k})^{\mathrm{op}} \rightarrow \text { Sets }
$$

such that for any scheme $S / k$,

(i) $\mathfrak{F}_{2}(S) \subset \mathfrak{F}(S)$.

(ii) Let $g_{i, i}: \mathscr{L}_{i} \rightarrow\left(V / V_{i}\right) \otimes \mathscr{O}_{S}$ be the sub-bundle morphism in Lemma 2.6 When $i \neq j$, set $g_{i, j}$ : $\mathscr{L}_{i} \rightarrow\left(V / V_{j}\right) \otimes \mathscr{O}_{S}$ to be the zero morphism. In this way, for any $I \subset[n]$ we get the following induced bundle morphism:

$$
g_{I}: \quad \mathscr{V}_{S} \bigoplus\left(\oplus_{i \in I} \mathscr{L}_{i}\right) \rightarrow \oplus_{i \in I}\left(V / V_{i}\right) \otimes \mathscr{O}_{S}
$$

For any $I \subset[n]$, we require $\bigwedge^{r+1-d_{I}+|I|} g_{I}=0$

Remark 2.2. - The Functor $\mathfrak{F}_{2}$ is represented by the scheme defined in Description II. $\mathfrak{F}$ is represented by $\Pi_{i=1}^{n} \mathbb{P}\left(V / V_{i}\right)$. Let the $n$ - tuple $\left(\mathscr{W}_{1}, \cdots, \mathscr{W}_{n}\right)$ be the universal $n$ - tuple of sub-bundles where for each $1 \leq i \leq n$, the vector bundle $\mathscr{W}_{i}$ is a sub-bundle of $V \otimes \mathscr{O}_{\mathbb{P}\left(V / V_{i}\right)}$ and contains $V_{i} \otimes \mathscr{O}_{\mathbb{P}\left(V / V_{i}\right)}$ as a co-rank one sub-bundle. By Lemma 2.6, $\mathscr{W}_{i} / V_{i} \otimes \mathscr{O}_{\mathbb{P}\left(V / V_{i}\right)}$ is $\mathscr{L}_{i}$. Thus on $\mathbb{P}\left(V / V_{i}\right)$, the induced morphism $0 \rightarrow \mathscr{L}_{i} \rightarrow\left(V / V_{i}\right) \otimes \mathscr{O}_{\mathbb{P}\left(V / V_{i}\right)}$ is the universal sub-line bundle on $\mathbb{P}\left(V / V_{i}\right)$. Plugging $\mathscr{L}_{i}$ 's in Condition (ii) in Definition 2.7 and giving coordinate systems for $V$ and for $V / V_{i}$ 's, which gives homogeneous coordinate of $\mathbb{P}\left(V / V_{i}\right)$, one gets the closed subscheme cut by the same homogeneous ideals as in Description II.

Now we arrive to the following comparison result:

Proposition 2.8. - The functors $\mathfrak{F}_{1}$ and $\mathfrak{F}_{2}$ are equal as sub-functors of $\mathfrak{F}$, thus Description I and II are equal as closed sub-schemes.

\section{Proof:}

In the whole proof, we will fix a scheme $S / k$. In order to compare the two functors, it suffices to compare on an open cover on $S$. For each point $p \in S / k$, there exits an affine open subscheme $U_{p}$ containing $p$, such that $\left.\left(\mathscr{W}_{1}, \cdots, \mathscr{W}_{n}\right)\right|_{U_{p}}$ is trivialized. For the rest of the argument, we fix such an open cover of $S$ over $k$. Henceforth we assume that the locally free sheaves $\mathscr{W}_{i}$ 's are trivialised. 
Since $\mathscr{V}_{S}$ and $\left.\left\{\mathscr{W}_{i}\right\}_{i=1}^{n}\right|_{U_{p}}$ are trivialized. Then we can represent $g_{I}$ as a matrix after choosing a basis for $\left.\mathscr{V}_{S}\right|_{U_{p}}$ and each $\left.\left(V / V_{i} \otimes \mathscr{O}_{S}\right)\right|_{U_{p}}$.

We can choose a basis for $\left.\mathscr{V}_{S}\right|_{U_{p}}$ and $\left.\left(V / V_{i}\right) \otimes \mathscr{O}_{S}\right|_{U_{p}}$, so that the quotient map $q_{i}:\left.\mathscr{V}_{S}\right|_{U_{p}} \rightarrow\left(V / V_{i}\right) \otimes$ $\left.\mathscr{O}_{S}\right|_{U_{p}}$ can be represented by the following matrix of the form: $\left[\begin{array}{c}A_{i} \\ b_{i}\end{array}\right]$, where $A_{i}$ is a $\left(r+1-d_{i}\right) \times(r+1)$ sub-matrix and $b_{i}$ a $1 \times(r+1)$ submatrix and furthermore the composed map $\left.q_{i}^{\prime \prime}\right|_{U_{p}}:\left.\mathscr{V}_{S}\right|_{U_{p}} \rightarrow\left(V / V_{i}\right) \otimes$ $\left.\left.\mathscr{O}_{S}\right|_{U_{p}} \rightarrow\left(\mathscr{V}_{S} / \mathscr{W}_{i}\right)\right|_{U_{p}}$ can be represented by $\left[A_{i}\right]$.

The natural sequence of maps $\left.\left.\left.\mathscr{W}_{i}\right|_{U_{p}} \hookrightarrow \mathscr{V}_{S}\right|_{U_{p}} \rightarrow\left(V / V_{i}\right) \otimes \mathscr{O}_{S}\right|_{U_{p}}$ factors though $h_{i}:\left.\mathscr{W}_{i}\right|_{U_{p}} /\left(V_{i} \otimes\right.$ $\left.\mathscr{O}_{S}\right)\left.\rightarrow\left(V / V_{i}\right) \otimes \mathscr{O}_{S}\right|_{U_{p}}$. Since $\left.\left(\mathscr{W}_{i} /\left(V_{i} \otimes \mathscr{O}_{S}\right)\right)\right|_{U_{p}}$ is a rank one module and the basis for $\left.\left(V / V_{i}\right) \otimes \mathscr{O}_{S}\right|_{U_{p}}$ has already been chosen, the matrix representation for $h_{i}$ is of the form : $\left[0 \cdots 0 \cdots a_{i}\right]^{T}$, which is a $1 \times\left(r+1-d_{i}\right)$ matrix. One can rescale the generator of $\left.\left(\mathscr{W}_{i} /\left(V_{i} \otimes \mathscr{O}_{S}\right)\right)\right|_{U_{p}}$ so that the matrix for $h_{i}$ is of the form: $[0 \cdots 0 \cdots 1]^{T}$.

The map $\left.f_{I}\right|_{U_{p}}:\left.\left.\mathscr{V}_{S}\right|_{U_{p}} \rightarrow \oplus_{i \in I}\left(\mathscr{V}_{S} / \mathscr{W}_{i}\right)\right|_{U_{p}}$ can be represented by the matrix of the form: $\left.f_{I}\right|_{U_{P}}=\left[\begin{array}{c}A_{i_{1}} \\ \vdots \\ A_{i_{\mid I I}}\end{array}\right]$, where $I=\left\{i_{1}, \cdots, i_{|I|}\right\}$.

The matrix representing $\left.g_{I}\right|_{U_{p}}$ is :

$$
\left.M_{I}\right|_{U_{p}}=\left[\begin{array}{ccccc}
A_{i_{1}} & 0 & & & \\
b_{i_{1}} & 1 & & & \\
\vdots & & & & \\
A_{i_{k}} & 0 & \ldots & 0 & \ldots \\
b_{i_{k}} & 0 & \ldots & 1 & \ldots \\
\vdots & & & & \\
A_{i_{|I|}} & \ldots & \ldots & \ldots & 0 \\
b_{i_{|I|}} & \ldots & \ldots & \ldots & 1
\end{array}\right]
$$

First we will show that $\mathfrak{F}_{1}$ is a subfunctor of $\mathfrak{F}_{2}$, i.e., for a scheme $S / k$, if $\left(\mathscr{W}_{1}, \cdots, \mathscr{W}_{n}\right) \in \mathfrak{F}_{1}(S)$, then it also has $\left(\mathscr{W}_{1}, \cdots, \mathscr{W}_{n}\right) \in \mathfrak{F}_{2}(S)$.

By condition (ii) the definition of $\mathfrak{F}_{1}$, we have $\left.\bigwedge^{r+1-d_{I}} f_{I}\right|_{U_{p}}=0$, i.e., every $\left(r+1-d_{I}\right) \times(r+$ $1-d_{I}$ ) minor of the matrix $\left[\begin{array}{c}A_{i_{1}} \\ \vdots \\ A_{i_{\mid I I}}\end{array}\right]$ is zero. If this is true, then by elementary linear algebra, every $\left(r+1-d_{I}+|I|\right) \times\left(r+1-d_{I}+|I|\right)$ minor of the matrix $\left.M_{I}\right|_{U_{p}}$ has to be zero.

Next, I claim that $\mathfrak{F}_{2}$ is a sub-functor of $\mathfrak{F}_{1}$.

By condition (ii) in the definition of $\mathfrak{F}_{2}$, at the every $\left(r+1-d_{I}+|I|\right) \times\left(r+1-d_{I}+|I|\right)$ minor of the matrix $\left.M_{I}\right|_{U_{p}}$ is zero, then we can choose $\left(r+1-d_{I}+|I|\right) \times\left(r+1-d_{I}+|I|\right)$ submatrix of $\left.g_{I}\right|_{U_{p}}$ 
with the following form

$$
\left[\begin{array}{cc}
W_{\left(r+1-d_{I}\right)} & 0 \\
0 & E_{|I|}
\end{array}\right]
$$

where $W_{\left(r+1-d_{I}\right)}$ is an arbitrary $\left(r+1-d_{I}\right) \times\left(r+1-d_{I}\right)$ submatrix of $\left.f_{I}\right|_{U_{p}}$ and $E_{|I|}$ is an $|I| \times|I|$ identity matrix. Then it implies that $\operatorname{det} W_{\left(r+1-d_{I}\right)}=0$, i.e., an arbitrary $\left(r+1-d_{I}\right) \times\left(r+1-d_{I}\right)$ minor of $\left.f_{I}\right|_{U_{p}}$ is zero. Then we conclude that $\mathfrak{F}_{2}$ is a sub-functor of $\mathfrak{F}_{1}$.

Therefore, we know that Description I ( i.e. $\mathscr{Z}_{1}$ ) and II (i.e. $\mathscr{Z}_{2}$ ) are equal as closed subschemes of $\Pi_{i=1}^{n} \mathbb{P}\left(V / V_{i}\right)$.

\section{MULTI-DEGREE OF THE CLOSURE OF THE IMAGE}

In this section, we will study the multi-degree of $X\left(V_{1}, \cdots, V_{n}\right)$. Recall that

$$
M(h)=\left\{\left(m_{1}, \cdots, m_{n}\right) \in \mathbb{Z}_{\geq 0}^{n}: \quad r+1-\sum_{i \in I} m_{i}>d_{I} \forall I \subset[n], \sum_{i=1}^{n} m_{i}=h\right\},
$$

and our main result of this section is :

Theorem 3.1. - Set $p=\max \{h ; M(h) \neq \emptyset\}$. The dimension of $X\left(V_{1}, \cdots, V_{n}\right)$ is $p$. Its multi-degree function takes value one at the integer vectors in $M(p)$ and zero otherwise.

Remark 3.2. - The closure of the image of the rational map is necessarily irreducible and reduced, and the moduli functor $\mathfrak{F}_{1}$ at least contains the closure of the image (actually, $\mathfrak{F}_{1}$ is the same as $X\left(V_{1}, \cdots, V_{n}\right)$, but we will prove this later). As long as we can show that the functor $\mathfrak{F}_{1}$ is multiplicity free and the closed points of $\mathfrak{F}_{1}$ are the closed the points of $X\left(V_{1}, \cdots, V_{n}\right)$ (by Theorem 2.1 ), the multi-degree of the closure of the image is the same as the multi-degree of $\mathfrak{F}_{1}$.

Recall that multi-degree of a closed subscheme of a product of projective spaces has a classical interpretation: for $\sum_{i=1}^{n} m_{i}=p$ and if there exists a general choice of $V^{i} \supset V_{i}$ with $\operatorname{codim} V^{i}=m_{i}$ in $V$ such that under the closed immersion $q_{c}: \Pi_{i=1}^{n} \mathbb{P}\left(V^{i} / V_{i}\right) \hookrightarrow \Pi_{i=1}^{n} \mathbb{P}\left(V / V_{i}\right)$, the closed subscheme $\Pi_{i=1}^{n} \mathbb{P}\left(V^{i} / V_{i}\right)$ intersects with $X\left(V_{1}, \cdots, V_{n}\right)$ at $k$ points counted with multiplicity, then the multi-degree function takes value $k$ at $\left(m_{1}, \ldots, m_{n}\right)$.

Before proving Theorem 3.1 , we need the following supporting proposition:

Proposition 3.1. - Given a $\left(c_{1}, \cdots, c_{n}\right) \in \mathbb{Z}_{\geq 0}^{n}$ with $r+1-\sum_{i \in I} c_{i}>d_{I}$, for any $I \subset[n]$, there exists a general choice of $V^{i} \supsetneqq V_{i}$ where $\operatorname{codim} V^{i}=c_{i}$ for each $i$, such that $\operatorname{dim} \cap_{i \in I} V^{i}=r+1-\sum_{i \in I} c_{i}$, for any $I \subset[n]$, and $\left.\operatorname{dim}\left(\cap_{i=1}^{n} V^{i}\right) \cap V_{k}\right)=\max _{I \subset[n]}\left\{\operatorname{dim}\left(\left(\cap_{i \in I} V_{i}\right) \cap V_{k}\right)-\sum_{i \notin I} c_{i}\right\}$ for each $1 \leq k \leq n$. In particular under this general choice of $\left\{V^{i}\right\}_{i=1}^{n}$, we have $\left(X\left(V_{1}, \cdots, V_{n}\right) \cap \Pi_{i=1}^{n} \mathbb{P}\left(V^{i} / V_{i}\right)\right) \neq \emptyset$. 
The next lemma is fundamental for the whole theory to the work out:

Lemma 3.2. - Let $\left\{c_{i}\right\}_{i=1}^{h}$ be a finite sequence of nonnegative integers and $V^{\prime}$ be a fixed subspace of $V$ with $\operatorname{dim} V=r+1$. If for any $I \subset[h]$, we have $\left(r+1-\sum_{i \in I} c_{i}\right)>\operatorname{dim}\left(\cap_{i \in I} V_{i}\right)$, then there exists $V^{i} \supset V_{i}$, for each $i \in[h]$ with $\operatorname{codim} V^{i}=c_{i}$, satisfying the following properties:

(i) $\operatorname{dim}\left(\cap_{i \in[h]} V^{i}\right)=r+1-\sum_{i \in[h]} c_{i}$,

(ii) $\operatorname{dim}\left(\cap_{i \in[h]} V^{i} \cap V^{\prime}\right) \leq \max _{I \subset[h]}\left\{\operatorname{dim} \cap_{i \in I} V_{i} \cap V^{\prime}-\sum_{i \notin I} c_{i}\right\}$.

Proof: This proof is by induction on the cardinality of $[h]$, when $h=1$, we can find $V^{1} \supset V_{1}$, with $\operatorname{codim} V^{1}=c_{1}$ and $\operatorname{dim}\left(V^{1} \cap V^{\prime}\right)=\max \left\{\operatorname{dim}\left(V^{\prime} \cap V_{1}\right), \operatorname{dim}\left(V^{\prime}\right)-c_{1}\right\}$ as follows:

Consider a decomposition $V=V^{\prime} \oplus V^{\prime \prime}$, such that $V_{1}=\left(V^{\prime} \cap V_{1}\right) \oplus\left(V^{\prime \prime} \cap V_{1}\right)$. Note that

$$
\operatorname{dim}\left(V^{\prime} \cap V_{1}\right) \leq \operatorname{dim}\left(V_{1}\right)<r+1-c_{1} .
$$

Consider another decomposition $V^{\prime \prime}=\left(V^{\prime \prime} \cap V_{1}\right) \oplus W_{(3)}$ and $V^{\prime}=\left(V^{\prime} \cap V_{1}\right) \oplus W_{(4)}$.

When $\operatorname{dim} W_{(3)} \geq\left(r+1-c_{1}-\operatorname{dim}\left(V_{1}\right)\right)$, then take a $\left(r+1-c_{1}-\operatorname{dim}\left(V_{1}\right)\right)$ dimensional subspace of $W_{(3)}$ and denote this subspace as $W_{(3)}^{\prime}$. Take $V_{1} \oplus W_{(3)}^{\prime}$ to be $V^{1}$, and then one can see that $\operatorname{dim}\left(V^{1} \cap\right.$ $\left.V^{\prime}\right)=\operatorname{dim}\left(V^{\prime} \cap V_{1}\right)$.

When $\operatorname{dim} W_{(3)}<\left(r+1-c_{1}-\operatorname{dim}\left(V_{1}\right)\right)$, note that $\operatorname{dim} W_{(3)}=r+1-\operatorname{dim} V^{\prime}-\left(\operatorname{dim} V_{1}-\operatorname{dim}\left(V^{\prime} \cap\right.\right.$ $\left.\left.V_{1}\right)\right)$, then we have $r+1-\operatorname{dim} V^{\prime}-\left(\operatorname{dim} V_{1}-\operatorname{dim}\left(V^{\prime} \cap V_{1}\right)\right)<r+1-c_{1}-\operatorname{dim}\left(V_{1}\right)$ thus $\operatorname{dim} V^{\prime}-c_{1}>$ $\operatorname{dim}\left(V_{1} \cap V^{\prime}\right)$. Take a $\operatorname{dim}\left(V^{\prime}\right)-c_{1}$ dimensional subspace of $V^{\prime}$ containing $V_{1} \cap V^{\prime}$ and denote this space as $W_{(4)}^{\prime}$, then $\operatorname{dim}\left(W_{(4)}^{\prime} \oplus V^{\prime \prime}\right)=\left(\operatorname{dim}\left(V^{\prime}\right)-c_{1}\right)+\left(r+1-\operatorname{dim} V^{\prime}\right)=r+1-c_{1}$. Take $W_{(4)}^{\prime} \oplus V^{\prime \prime}$ to be $V^{1}$, and one can see that $\operatorname{dim}\left(V^{1} \cap V^{\prime}\right)=\operatorname{dim}\left(V^{\prime}\right)-c_{1}$. Thus we conclude that there exists $V^{1}$ with $\operatorname{codim} V^{1}=c_{1}$ and $\operatorname{dim}\left(V^{1} \cap V^{\prime}\right)=\max \left\{\operatorname{dim}\left(V^{\prime} \cap V_{1}\right), \operatorname{dim}\left(V^{\prime}\right)-c_{1}\right\}$.

When $h \leq k$, assume the lemma is true. By the assumption, there exists a system of linear subspaces of $\left\{V^{i}\right\}_{i=1}^{k}$ satisfying the properties (i) and (ii) with respect to each of $V_{k+1}, V^{\prime}$ or $\left(V_{k+1} \cap V^{\prime}\right)$.

Let $\operatorname{Gr}\left(c_{i}, V / V_{i}\right)$ be the irreducible moduli scheme parameterizing all codimension $c_{i}$ subspaces of $V$ containing $V_{i}$. The subset of $\prod_{i=1}^{k} \operatorname{Gr}\left(c_{i}, V / V_{i}\right)$ satisfying Properties (i) and (ii) with respect to all $V_{k+1}, V^{\prime}$ and $\left(V_{k+1} \cap V^{\prime}\right)$ is the intersection of three non-empty open subset of $\Pi_{i=1}^{k} \operatorname{Gr}\left(c_{i}, V / V_{i}\right)$, which is irreducible, so each open subset of this moduli space is open and dense. Note that intersection of three dense nonempty open subsets is nonempty, so there exists a system of linear subspaces $\left\{V^{i}\right\}_{i=1}^{k}$ satisfying Properties (i) and (ii) with respect to both $V_{k+1}, V^{\prime}$ and $V_{k+1} \cap V^{\prime}$, and for the rest of the proof, we will fix such a system $\left\{V^{i}\right\}_{i=1}^{k}$.

First, I claim that there exists $V^{k+1} \supset V_{k+1}$ with $\operatorname{codim} V^{k+1}=c_{k+1}$, such that $\operatorname{dim} \cap_{i=1}^{k+1} V^{i}=r+1-$ $\sum_{i=1}^{k+1} c_{i}$. We can construct such a $V^{k+1}$ as follows: 
Consider a decomposition $V=\cap_{i=1}^{k} V^{i} \oplus V^{K}$, such that

$$
V_{k+1}=\left(\left(\cap_{i=1}^{k} V^{i}\right) \bigcap V_{k+1}\right) \oplus\left(V^{K} \cap V_{k+1}\right) .
$$

Then $\operatorname{dim} V^{K}=(r+1)-\left(r+1-\sum_{i=1}^{k} c_{i}\right)=\sum_{i=1}^{k} c_{i}$, but $\operatorname{dim} V^{k+1}=r+1-c_{k+1}>\sum_{i=1}^{k} c_{i}$ (because $\left.r+1-\sum_{i=1}^{k+1} c_{i}>\operatorname{dim}\left(\cap_{i=1}^{k+1} V_{i}\right)\right)$.

$\operatorname{dim}\left(\cap_{i=1}^{k} V^{i}\right)=r+1-\sum_{i=1}^{k} c_{i} \geq r+1-\sum_{i=1}^{k+1} c_{i}$, and also notice that for any $I \subset[k]$, we have

$$
\begin{aligned}
\operatorname{dim}\left(\cap_{i \in I} V_{i} \bigcap V_{k+1}\right)-\sum_{i \notin I, i \in[k]} c_{i}< & r+1-\sum_{I \cup\{k+1\}} c_{i}-\sum_{i \notin I, i \in[k]} c_{i} \\
& =r+1-\sum_{i=1}^{k+1} c_{i}
\end{aligned}
$$

Therefore,

$$
\operatorname{dim}\left(\cap_{i=1}^{k} V^{i} \bigcap V_{k+1}\right) \leq \max _{I \subset[k]}\left\{\operatorname{dim}\left(\cap_{i \in I} V_{i} \bigcap V_{k+1}\right)-\sum_{i \notin I} c_{i}\right\}<r+1-\sum_{i=1}^{k+1} c_{i}
$$

then take a $\left(r+1-\sum_{i=1}^{k+1} c_{i}\right)$ dimensional subspace of $\cap_{i=1}^{k} V^{i}$ containing $\cap_{i=1}^{k} V^{i} \cap V_{k+1}$, and denote this subspace as $V_{[k]}^{\prime}$.

$$
\begin{aligned}
\operatorname{dim}\left(V^{K} \oplus V_{[k]}^{\prime}\right) & =\operatorname{dim}\left(V^{K}\right)+\operatorname{dim} V_{[k]}^{\prime} \\
& =\sum_{i=1}^{k} c_{i}+\left(r+1-\sum_{i=1}^{k+1} c_{i}\right) \\
& =r+1-c_{k+1},
\end{aligned}
$$

then take $V^{k+1}=V^{K} \oplus V_{[k]}^{\prime}$. Then this $V^{k+1}$ has the property that $V^{k+1} \supset V_{k+1}$ with codimension $c_{k}$ and $\operatorname{dim}\left(V^{k+1} \cap \cap_{i=1}^{k} V^{i}\right)=r+1-\sum_{i=1}^{k+1} c_{i}$, which is the minimal intersection dimension of $V^{i}$, satisfying $\operatorname{codim} V^{i}=c_{i}$.

Secondly, I claim that we can construct a $V^{k+1}$ with the property

$$
\operatorname{dim}\left(\cap_{i=1}^{k+1} V^{i} \bigcap V^{\prime}\right) \leq \max _{I \subset[k+1]}\left\{\operatorname{dim}\left(\cap_{i \in I} V_{i} \bigcap V^{\prime}\right)-\sum_{i \notin I[k+1]} c_{i}\right\}
$$

Consider a decomposition $V=\left(\cap_{i=1}^{k} V^{k} \cap V^{\prime}\right) \oplus W$ such that $V_{k+1}=\left(\cap_{i=1}^{k} V^{k} \cap V^{\prime} \cap V_{k+1}\right) \oplus(W \cap$ $\left.V_{k+1}\right)$.

When $\operatorname{dim} W \geq r+1-c_{k+1}-\operatorname{dim}\left(\cap_{i=1}^{k} V^{i} \cap V^{\prime} \cap V_{k+1}\right)$, take $\left[r+1-c_{k+1}-\operatorname{dim}\left(\cap_{i=1}^{k} V^{i} \cap V^{\prime} \cap V_{k+1}\right)\right]$ dimensional subspace of $W$ containing $W \cap V_{k+1}$ (one can do this because $\operatorname{dim}\left(W \cap V_{k+1}\right)+$ $\operatorname{dim}\left(\cap_{i=1}^{k} V^{i} \cap V^{\prime} \cap V_{k+1}\right)=\operatorname{dim}\left(V_{k+1}\right)$ and $\left.\operatorname{dim} V_{k+1}<r+1-c_{k+1}\right)$ and denote this subspace as $W^{\prime}$. Take $V^{k+1}$ to be $\operatorname{Span}\left(V_{k+1}, W^{\prime}\right)$. One can check that $\operatorname{dim}\left(\operatorname{Span}\left(V_{k+1}, W^{\prime}\right)\right)=r+1-c_{k+1}$, and $\operatorname{dim}\left(\operatorname{Span}\left(V_{k+1}, W^{\prime}\right) \cap\left(\cap_{i=1}^{k} V^{i} \cap V^{\prime}\right)\right)=\operatorname{dim}\left(V_{k+1} \cap\left(\cap_{i=1}^{k} V^{i} \cap V^{\prime}\right)\right)$. Then in this case there exists $V^{k+1}$, such that $\operatorname{dim}\left(V^{k+1} \cap\left(\cap_{i=1}^{k} V^{i} \cap V^{\prime}\right)\right) \leq \max \left\{\operatorname{dim}\left(\cap_{i=1}^{k} V^{i} \cap V^{\prime} \cap V_{k+1}\right), \operatorname{dim}\left(\cap_{i=1}^{k} V^{i} \cap V^{\prime}\right)-\right.$ $\left.\left.c_{k+1}\right)\right\}$. 
When $\operatorname{dim} W<r+1-c_{k+1}-\operatorname{dim}\left(\cap_{i=1}^{k} V^{i} \cap V^{\prime} \cap V_{k+1}\right)$, note that $\operatorname{dim} W=r+1-\operatorname{dim}\left(\cap_{i=1}^{k} V^{i} \cap V^{\prime}\right)$, then we have

$$
r+1-\operatorname{dim}\left(\cap_{i=1}^{k} V^{i} \bigcap V^{\prime}\right)<r+1-c_{k+1}-\operatorname{dim}\left(\cap_{i=1}^{k} V^{i} \bigcap V^{\prime} \bigcap V_{k+1}\right),
$$

thus $\operatorname{dim}\left(\cap_{i=1}^{k} V^{i} \cap V^{\prime} \cap V_{k+1}\right)<\operatorname{dim}\left(\cap_{i=1}^{k} V^{i} \cap V^{\prime}\right)-c_{k+1}$. Take $\operatorname{dim}\left(\cap_{i=1}^{k} V^{i} \cap V^{\prime}\right)-c_{k+1} \operatorname{dimensional}$ linear subspace of $\cap_{i=1}^{k} V^{i} \cap V^{\prime}$ containing $\cap_{i=1}^{k} V^{i} \cap V^{\prime} \cap V_{k+1}$ and denote this linear space as $W^{\prime \prime}$. Notice that

$$
\begin{aligned}
\operatorname{dim}\left(W^{\prime \prime} \oplus W\right) & =\left(\operatorname{dim}\left(\cap_{i=1}^{k} V^{i} \cap V^{\prime}\right)\right)-c_{k+1}+\left(r+1-\operatorname{dim}\left(\cap_{i=1}^{k} V^{i} \cap V^{\prime}\right)\right) . \\
& =r+1-c_{k+1}
\end{aligned}
$$

Also $\left.\operatorname{dim}\left(\left(W^{\prime \prime} \oplus W\right) \bigcap\left(\cap_{i=1}^{k} V^{i} \cap V^{\prime}\right)\right)=\operatorname{dim}\left(W^{\prime \prime} \cap\left(\cap_{i=1}^{k} V^{i} \cap V^{\prime}\right)\right)\right)=\operatorname{dim}\left(\cap_{i=1}^{k} V^{i} \cap V^{\prime}\right)-c_{k+1}$. Take $V^{k+1}$ to be $W^{\prime \prime} \oplus W$, Then in this case there exists $V^{k+1}$, such that $\operatorname{dim}\left(V^{k+1} \cap\left(\cap_{i=1}^{k} V^{i} \cap V^{\prime}\right)\right) \leq$ $\left.\max \left\{\operatorname{dim}\left(\cap_{i=1}^{k} V^{i} \cap V^{\prime} \cap V_{k+1}\right), \operatorname{dim}\left(\cap_{i=1}^{k} V^{i} \cap V^{\prime}\right)-c_{k+1}\right)\right\}$.

Combining the above two cases, we have proved that there always exists $V^{k+1}$ whose codimension is $c_{k+1}$ such that the intersection dimension of $V^{k+1}$ with $\cap_{i=1}^{k} V^{i} \cap V^{\prime}$ is not bigger than

$$
\left.\max \left\{\operatorname{dim}\left(\cap_{i=1}^{k} V^{i} \bigcap V^{\prime} \cap V_{k+1}\right), \operatorname{dim}\left(\cap_{i=1}^{k} V^{i} \bigcap V^{\prime}\right)-c_{k+1}\right)\right\}
$$

Recall that

$$
\operatorname{dim}\left(\cap_{i=1}^{k} V^{i} \bigcap V^{\prime} \cap V_{k+1}\right) \leq \max _{I \subset[k]}\left\{\operatorname{dim}\left(\cap_{i \in I} V_{i} \bigcap\left(V^{\prime} \cap V_{k+1}\right)\right)-\sum_{i \in[k] \backslash I} c_{i}\right\}
$$

therefore we have:

$$
\begin{aligned}
& \max \left\{\operatorname{dim}\left(\cap_{i=1}^{k} V^{i} \cap V^{\prime} \cap V_{k+1}\right), \operatorname{dim}\left(\cap_{i=1}^{k} V^{i} \cap V^{\prime}\right)-c_{k+1}\right\} \\
\leq & \max _{I \subset[k]}\left\{\operatorname{dim}\left(\cap_{i \in I} V_{i} \cap\left(V^{\prime} \cap V_{k+1}\right)\right)-\sum_{i \in[k] \backslash I} c_{i},\left(\operatorname{dim} \cap_{i \in I} V_{i} \cap V^{\prime}-\sum_{i \in[k] \backslash I} c_{i}\right)-c_{k+1}\right\} \\
= & \max _{I \subset[k+1]}\left\{\operatorname{dim}\left(\cap_{i \in I} V_{i} \cap V^{\prime}\right)-\sum_{i \in[k+1] \backslash I} c_{i}\right\}
\end{aligned}
$$

Therefore, all of the possible $V^{k+1}$ 's satisfying Case (i) form an nonempty open subset on $\operatorname{Gr}\left(c_{k+1}, V / V_{k+1}\right)$, where $\operatorname{Gr}\left(c_{k+1}, V / V_{k+1}\right)$ is the irreducible moduli scheme parameterizing all of the codimension $c_{k+1}$ linear subspaces in $V$ containing $V_{k+1}$. All of the possible $V^{k+1}$ satisfying Case (ii) also form an nonempty open subset on $\operatorname{Gr}\left(c_{k+1}, V / V_{k+1}\right)$. So the all of the possible $V^{k+1}$ satisfying both Cases (i) and (ii) form the intersection of two nonempty open subsets of the $\operatorname{Gr}\left(c_{k+1}, V / V_{k+1}\right)$, which is still open and dense because $\operatorname{Gr}\left(c_{k+1}, V / V_{k+1}\right)$ is irreducible, thus nonempty, so there exists one $V^{k+1}$ satisfying both Cases (i) and (ii).

Thus we have found $\left\{V^{i}\right\}_{i=1}^{k+1}$ satisfying Properties (i) and (ii). Then the lemma follows.

Remark 3.3. - A priori, given $V^{i} \supset V_{i}$ with $\operatorname{codim} V^{i}=c_{i}$ for each $i$, we always have $\operatorname{dim}\left(\cap_{i=1}^{n} V^{i} \cap V^{\prime}\right) \geq$ $\operatorname{dim}\left(\cap_{i \in I} V_{i} \cap V^{\prime}\right)-\sum_{i \notin I} c_{i}$ for any $I \subset[n]$, therefore $\max _{I \subset[h]}\left\{\operatorname{dim} \cap_{i \in I} V_{i} \cap V^{\prime}-\sum_{i \notin I} c_{i}\right\}$ in the Lemma 3.2 is the actual minimal possible intersection dimension of $\bigcap_{i \in[n]} V^{i}$ with $V^{\prime}$ 
Now we are in a good position to prove Proposition 3.1

\section{Proof of Proposition 3.1.}

Take $V^{\prime}=V_{k}, 1 \leq k \leq n$, then by Lemma 3.2, we can find a sequence $\left\{V^{i} \supset V_{i}\right\}_{i=1}^{n}$, such that $\operatorname{dim}\left(\cap_{i \in I} V^{i}\right)=r+1-\sum_{i \in I} c_{i}$ for any $I \subset[n]$, also $\operatorname{dim}\left(\left(\cap_{i=1}^{n} V^{i}\right) \cap V_{k}\right) \leq \max _{I \subset[n]}\left\{\operatorname{dim}\left(\left(\cap_{i \in I} V_{i}\right) \cap V_{k}\right)-\right.$ $\left.\sum_{i \in[n] \backslash I} c_{i}\right\}$. By Remark 3.3, $\max _{I \subset[h]}\left\{\operatorname{dim} \cap_{i \in I} V_{i} \cap V_{k}-\sum_{i \notin I} c_{i}\right\}$ in the Lemma 3.2 is the actual minimal possible intersection dimension of $\bigcap_{i \in[n]} V^{i}$ with $V_{k}$.

Note that $\left\{V^{i}\right\}_{i=1}^{n}$ with codim $V^{i}=c_{i}$ for each $i$ corresponds to a point in the following irreducible moduli scheme $\Pi_{i=1}^{n} \operatorname{Gr}\left(c_{i}, V / V_{i}\right)$ where $\operatorname{Gr}\left(c_{i}, V / V_{i}\right)$ is the moduli scheme parameterizing all of codimension $c_{i}$ linear subspaces containing $V_{i}$. The condition $\operatorname{dim} \cap_{i \in I} V^{i}=r+1-\sum_{i \in I} c_{i}$ for any $I \subset[n]$ is a nonempty open condition and also $\operatorname{dim}\left(\cap_{i=1}^{n} V^{i}=1\right) \cap V_{k}=\max _{I \subset[h]}\left\{\operatorname{dim} \cap_{i \in I} V_{i} \cap V_{k}-\sum_{i \notin I} c_{i}\right\}$ is also a nonempty open condition by the above discussion. Therefore for each $k \in[n]$, satisfying $\operatorname{dim} \cap_{i \in I} V^{i}=r+1-\sum_{i \in I} c_{i}$ and $\operatorname{dim}\left(\cap_{i=1}^{n} V^{i}=1\right) \cap V_{k}=\max _{I \subset[h]}\left\{\operatorname{dim} \cap_{i \in I} V_{i} \cap V_{k}-\sum_{i \notin I} c_{i}\right\}$ is the intersection of two nonempty open dense subsets of the moduli scheme, which is still a nonempty open condition in $\Pi_{i=1}^{n} \operatorname{Gr}\left(c_{i}, V / V_{i}\right)$, and we denote this nonempty open set of $\Pi_{i=1}^{n} \operatorname{Gr}\left(c_{i}, V / V_{i}\right)$ as $U_{k}$.

Then we take $\cap_{i=1}^{n} U_{k}$ is still nonempty and open, thus we can take a point in this nonempty set, which corresponds to $\left\{V^{i}\right\}_{i=1}^{n}$ with $\operatorname{dim}\left(\cap_{i \in I} V^{i}\right)=r+1-\sum_{i \in I} c_{i}$ and $\operatorname{dim}\left(\left(\cap_{i=1}^{n} V^{i}\right) \cap\left(V_{k}\right)\right)=$ $\max _{I \subset[h]}\left\{\operatorname{dim} \cap_{i \in I} V_{i} \cap V_{k}-\sum_{i \notin I} c_{i}\right\}$.

Since $d_{I}<r+1-\sum_{i \in I} c_{i}$, for any $I \subset[n]$, therefore

$$
\begin{aligned}
\operatorname{dim}\left(\left(\cap_{i \in[n]} V^{i}\right) \cap V_{k}\right) & =\max _{I \subset[n]}\left\{\operatorname{dim}\left(\left(\cap_{i \in I} V_{i}\right) \cap V_{k}\right)-\sum_{i \notin I} c_{i}\right\} \\
& <r+1-\sum_{i=1}^{n} c_{i} \\
& =\operatorname{dim} \bigcap_{i=1}^{n} V^{i} .
\end{aligned}
$$

Then for each $i$, there exists a vector $v_{i} \in \cap_{j \in[n]} V^{j}$ such that $v_{i} \notin V_{i}$, then there exists a vector $v_{[n]} \in \cap_{i \in[n]} V^{i}$ such that $v_{[n]} \notin V_{i}$ for each $i$. Therefore, the intersection of $\Pi_{i=1}^{n} \mathbb{P}\left(V^{i} / V_{i}\right)$ with $X\left(V_{1}, \cdots, V_{n}\right)$ contains a point $\left(\operatorname{Span}\left(V_{1}, v_{[n]}\right), \cdots, \operatorname{Span}\left(V_{n}, v_{[n]}\right)\right)$, which implies for such a general choice of $\left\{V^{i}\right\}_{i=1}^{n}$, the intersection is not empty.

The following lemma is used to detect at which integer vector the multi-degree function takes zero.

Lemma 3.3. - Given an integer vector $\left(c_{1}, \cdots, c_{n}\right)$ such that there exists $I \subset[n]$ with $r+1-\sum_{i \in I} c_{i} \leq$ $\operatorname{dim}\left(\cap_{i \in I} V_{i}\right)$, then there exists $\Pi_{i=1}^{n} \mathbb{P}^{r+1-d_{i}-c_{i}} \hookrightarrow \Pi_{i=1} \mathbb{P}\left(V / V_{i}\right)$,such that it has empty intersection with $X\left(V_{1}, \cdots, V_{n}\right)$..

\section{Proof:}


Rational points of $\Pi_{i=1}^{n} \mathbb{P}^{r+1-d_{i}-c_{i}} \hookrightarrow \Pi_{i=1} \mathbb{P}\left(V / V_{i}\right)$ correspond to $\left(V^{1}, \cdots, V^{n}\right)$ with $V^{i} \supsetneqq V_{i}$ and $\operatorname{codim} V^{i}=c_{i}$. If we want to show there exists $\Pi_{i=1}^{n} \mathbb{P}^{r+1-d_{i}-c_{i}} \hookrightarrow \Pi_{i=1} \mathbb{P}\left(V / V_{i}\right)$ with empty intersection with the closure of the rational map $\mathbb{P}(V) \rightarrow \mathbb{P}\left(V / V_{i}\right)$, all we need to do is to construct a system of $\left\{V^{i} \supset V_{i}\right\}_{i=1}^{n}$ such that it does not contain $\left(W_{1}, \ldots, W_{n}\right)$ with $\forall I \subset[n], \cap_{i \in I} W_{i} \supsetneqq \cap_{i \in I} V_{i}$.

First observe that since there exists $I \subset[n]$ with $r+1-\sum_{i \in I} c_{i} \leq \operatorname{dim}\left(\cap_{i \in I} V_{i}\right)$, then there exists $I^{\prime} \subset I$, such that for any $I^{\prime \prime} \varsubsetneqq I^{\prime}, r+1-\sum_{i \in I^{\prime \prime}} c_{i}>\operatorname{dim}\left(\cap_{i \in I^{\prime \prime}} V_{i}\right)$ but also $r+1-\sum_{i \in I^{\prime}} c_{i} \leq \operatorname{dim}\left(\cap_{i \in I^{\prime}} V_{i}\right)$.

When $\left|I^{\prime}\right|=1$, we have $r+1-c_{I^{\prime}} \leq d_{I^{\prime}}$. In this case, any linear subspace of $V$ strictly containing $V_{I^{\prime}}$ has codimension less than $c_{I^{\prime}}$. Thus any $\Pi_{i=1}^{n} \mathbb{P}^{r+1-d_{i}-c_{i}} \hookrightarrow \Pi_{i=1} \mathbb{P}\left(V / V_{i}\right)$ has empty intersection with $X\left(V_{1}, \cdots, V_{n}\right)$.

When $\left|I^{\prime}\right|>1$, we will construct $\left\{V^{i}\right\}_{i \in[n]}$ with $\operatorname{codim} V^{i}=c_{i}, \forall i \in[n]$ such that there exists $I^{\prime} \subset[n]$ with $\operatorname{dim}\left(\cap_{i \in I^{\prime}} V^{i}\right)=\operatorname{dim}\left(\cap_{i \in I^{\prime}} V_{i}\right)$. Let $i^{\prime \prime} \in I^{\prime}$, consider $I^{\prime} \backslash i^{\prime \prime}$, then by Lemma 3.2, there exists $\left\{V^{i} \supset\right.$ $\left.V_{i}\right\}_{i \in I^{\prime} \backslash i^{\prime \prime}}$, with $\operatorname{dim}\left(\cap_{i \in I^{\prime} \backslash i^{\prime \prime}} V^{i}\right)=r+1-\sum_{i \in I^{\prime} \backslash i^{\prime \prime}} c_{i}$ and also

$$
\operatorname{dim}\left(\cap_{i \in I^{\prime} \backslash i^{\prime \prime}} V^{i}\right) \bigcap V_{i^{\prime \prime}} \leq \max _{\mathscr{K} \subset I^{\prime \prime}}\left\{\operatorname{dim}\left(\cap \mathscr{K} \subset I^{\prime \prime} V_{i} \bigcap V_{i^{\prime \prime}}\right)-\sum_{i \in I^{\prime \prime} \backslash \mathscr{K}} c_{i}\right\},
$$

where $I^{\prime \prime}$ is $I^{\prime} \backslash i^{\prime \prime}$.

I claim that $\operatorname{dim}\left(\cap_{i \in I^{\prime \prime}} V^{i}\right) \bigcap V_{i^{\prime \prime}}=\operatorname{dim}\left(\cap_{i \in I^{\prime}} V_{i}\right)$.

First let's prove that $\max _{\mathscr{K} \subset I^{\prime \prime}}\left\{\operatorname{dim}\left(\cap \mathscr{K} \subset I^{\prime \prime} V_{i} \cap V_{i^{\prime \prime}}\right)-\sum_{i \in I^{\prime \prime} \backslash \mathscr{K}} c_{i}\right\}=\operatorname{dim}\left(\cap_{i \in I^{\prime}} V_{i}\right)$.

When $\mathscr{K} \varsubsetneqq I^{\prime \prime}$, we have $\mathscr{K} \cup\left\{i^{\prime \prime}\right\} \varsubsetneqq I^{\prime}$, then

$$
\begin{aligned}
\operatorname{dim}\left(\cap_{i \in \mathscr{K} \subset I^{\prime \prime}} V_{i} \cap V_{i^{\prime \prime}}\right)-\sum_{i \in I^{\prime \prime} \backslash \mathscr{K}} c_{i} & \leq\left(r+1-\sum_{\mathscr{K} \cup\left\{i^{\prime \prime}\right\}} c_{i}\right)-\sum_{i \in I^{\prime \prime} \backslash \mathscr{K}} c_{i} \\
& =r+1-\sum_{i \in I^{\prime}} c_{i} \\
\leq & \operatorname{dim}\left(\cap_{i \in I^{\prime}} V_{i}\right)
\end{aligned}
$$

When $\mathscr{K}=I^{\prime \prime}$, we have $\operatorname{dim}\left(\cap \mathscr{K} \subset I^{\prime \prime} V_{i} \cap V_{i^{\prime \prime}}\right)-\sum_{i \in I^{\prime \prime} \backslash \mathscr{K}} c_{i}=\operatorname{dim}\left(\cap_{i \in I^{\prime}} V_{i}\right)$. Thus it follows that $\max _{\mathscr{K} \subset I^{\prime \prime}}\left\{\operatorname{dim}\left(\cap \mathscr{K} \subset I^{\prime \prime} V_{i} \cap V_{i^{\prime \prime}}\right)-\sum_{i \in I^{\prime \prime} \backslash \mathscr{K}} c_{i}\right\}=\operatorname{dim}\left(\cap_{i \in I^{\prime}} V_{i}\right)$. Therefore $\operatorname{dim}\left(\cap_{i \in I^{\prime \prime}} V^{i}\right) \cap V_{i^{\prime \prime}} \leq$ $\operatorname{dim}\left(\cap_{i \in I^{\prime}} V_{i}\right)$

But since $V^{i} \supset V_{i}$, we have $\operatorname{dim}\left(\cap_{i \in I^{\prime \prime}} V^{i}\right) \cap V_{i^{\prime \prime}} \geq \operatorname{dim}\left(\cap_{i \in I^{\prime}} V_{i}\right)$. Then we have $\operatorname{dim}\left(\cap_{i \in I^{\prime \prime}} V^{i}\right) \bigcap V_{i^{\prime \prime}}=$ $\operatorname{dim}\left(\cap_{i \in I^{\prime}} V_{i}\right)$, thus the claim follows.

Now consider a decomposition $V=\cap_{i \in I^{\prime \prime}} V^{i} \oplus V^{D}$ such that $V_{i^{\prime \prime}}=\left(\left(\cap_{i \in I^{\prime \prime}} V^{i}\right) \cap V_{i^{\prime \prime}}\right) \oplus\left(V^{D} \cap V_{i^{\prime \prime}}\right)$. Note that $\operatorname{dim}\left(V^{D}\right)=(r+1)-\left(r+1-\sum_{i \in I^{\prime \prime}} c_{i}\right)=\sum_{i \in I^{\prime \prime}} c_{i} \geq r+1-c_{i^{\prime \prime}}-\operatorname{dim} \cap_{i \in I^{\prime \prime}} V^{i} \cap V_{i^{\prime \prime}}$, so let's take $\left(r+1-c_{i^{\prime \prime}}-\operatorname{dim} \cap \cap_{i \in I^{\prime \prime}} V^{i} \cap V_{i^{\prime \prime}}\right)$-dimensional subspace $V^{d}$ of $V^{D}$ such that $V^{d} \supset V_{i^{\prime \prime}} \cap V^{D}$ and take $V^{i^{\prime \prime}}=\operatorname{Span}\left(V_{i^{\prime \prime}}, V^{d}\right)$. (note that $\left.\operatorname{dim}\left(V_{i^{\prime \prime}} \cap V^{D}\right)+\operatorname{dim}\left(\cap_{i \in I^{\prime \prime}} V^{i} \cap V_{i^{\prime \prime}}\right)=\operatorname{dim} V_{i^{\prime \prime}}<r+1-c_{i^{\prime \prime}}\right)$.

Therefore, we have proved that there exists $\left\{V^{i} \supset V_{i}\right\}_{i \in I^{\prime}}$ with $\operatorname{codim} V^{i}=c_{i}$ and $\operatorname{dim}\left(\cap_{i \in I^{\prime}} V^{i}\right)=$ $\operatorname{dim} \cap_{i \in I^{\prime}} V_{i}$.

Also, points of the closure of the rational map $\mathbb{P}(V) \rightarrow \mathbb{P}\left(V / V_{i}\right)$ correspond to $\left(W_{1}, \cdots, W_{n}\right)$ with $W_{i}$ a linear subspace of $V$ containing $V_{i}$ as a codimension one subspace, such that $\forall \mathscr{K} \subset$ 
$[n], \operatorname{dim}\left(\cap_{i \in \mathscr{K}} W_{i}\right)>\operatorname{dim}\left(\cap_{i \in \mathscr{K}} V_{i}\right)$. Then the $\left\{V^{i}\right\}_{i=1}^{n}$ satisfying for a $I^{\prime} \subset[n], \operatorname{dim}\left(\cap_{i \in I^{\prime}} V^{i}\right)=$ $\operatorname{dim} \cap_{i \in I}\left(V_{i}\right)$, does not contain any $\left(W_{1}, \cdots, W_{n}\right)$ because $\operatorname{dim}\left(\cap_{i \in I^{\prime}} W_{i}\right)>\operatorname{dim}\left(\cap_{i \in I^{\prime}} V_{i}\right)$. Thus the lemma follows.

Proposition 3.4. - The dimension of the image of the rational map:

$$
\mathbb{P}(V) \rightarrow \Pi_{i=1}^{n} \mathbb{P}\left(V / V_{i}\right), \bigcap_{i=1}^{n} V_{i}=\{0\}
$$

is the largest $p$ such that $M(p) \neq \emptyset$. And its multi-degree function takes a nonzero value at the integer vectors in $M(p)$ and zero otherwise.

Proof: In this proof, $t_{i}=\operatorname{dim}\left(V / V_{i}\right)-1$ for each $1 \leq i \leq n$, so for the rest of the proof, we identify $\mathbb{P}\left(V / V_{i}\right)$ with $\mathbb{P}^{t_{i}}$. Given an ample divisor $\sum_{i=1}^{n} c_{i} \mathbb{P}^{t_{1}} \times \cdots \times \mathbb{P}^{t_{i}-1} \times \cdots \times \mathbb{P}^{t_{n}}$ in $\Pi_{i=1}^{n} \mathbb{P}^{t_{i}} \hookrightarrow \mathbb{P}^{\ell}$, where $c_{i} \gg 0$ for each $1 \leq i \leq n$, we get a closed immersion:

$$
f_{c}: \quad \prod_{i=1}^{n} \mathbb{P}^{t_{i}} \hookrightarrow \mathbb{P}^{\ell}
$$

Let $D(p)=\left\{\left(u_{1}, \cdots, u_{n}\right) \in \mathbb{Z}_{\geq 0}^{n}: \sum_{i=1}^{n} u_{i}=p\right\}$. I claim that in Chow ring of $\Pi_{i=1}^{n} \mathbb{P}^{m_{i}}$, the class $\left(\sum_{i=1}^{n} c_{i} \mathbb{P}^{t_{1}} \times \cdots \times \mathbb{P}^{t_{i}-1} \times \cdots \times \mathbb{P}^{t_{n}}\right)^{k}$ where $u_{i} \leq t_{i}$ for each $1 \leq i \leq n$, can be represented by the form $\sum_{\left(u_{1}, \cdots, u_{n}\right) \in D(k)} c_{\left(u_{1}, \cdots, u_{n}\right)} \Pi_{i=1}^{n} \mathbb{P}^{t_{i}-u_{i}}$ where all $c_{\left(u_{1}, \cdots, u_{n}\right)}$ are positive. One can prove this by induction. Assume that when $h=k$ it is correct. Let $e_{j}=(0, \cdots, 1, \cdots, 0)$ whose $j$-th component is one and other components are zero. When $h=k+1$,

$$
\begin{aligned}
& \left(\sum_{i=1}^{n} c_{i} \mathbb{P}^{t_{1}} \times \cdots \mathbb{P}^{t_{i}-1} \cdots \mathbb{P}^{t_{n}}\right)^{k+1} \\
= & \left(\sum_{i=1}^{n} c_{i} \mathbb{P}^{t_{1}} \times \cdots \mathbb{P}^{t_{i}-1} \cdots \mathbb{P}^{t_{n}}\right)^{k} \cdot\left(\sum_{i=1}^{n} c_{i} \mathbb{P}^{t_{1}} \times \cdots \times \mathbb{P}^{t_{i}-1} \times \cdots \times \mathbb{P}^{t_{n}}\right) \\
= & \left(\sum_{\left(u_{1}, \cdots, u_{n}\right) \in D(k)} c_{\left(u_{1}, \cdots, u_{n}\right)} \Pi_{i=1}^{n} \mathbb{P}^{t_{i}-u_{i}}\right) \cdot\left(\sum_{i=1}^{n} c_{i} \mathbb{P}^{t_{1}} \times \cdots \times \mathbb{P}^{t_{i}-1} \times \cdots \times \mathbb{P}^{t_{n}}\right) \\
= & \left(\sum_{\left(u_{1}, \cdots, u_{n}\right) \in D(k)} c_{\left(u_{1}, \cdots, u_{n}\right)} \Pi_{i=1}^{n} \mathbb{P}^{t_{i}-u_{i}}\right) \cdot\left(\sum_{i=1}^{n} c_{i} \mathbb{P}^{t_{1}} \times \cdots \times \mathbb{P}^{t_{i}-1} \times \cdots \times \mathbb{P}^{t_{n}}\right) \\
= & \left(\sum_{\left(u_{1}, \cdots, u_{n}\right) \in D(k)} c_{\left(u_{1}, \cdots, u_{n}\right)} \sum_{j=1}^{n}\left(c_{j} \Pi_{i \in[n \backslash j} \mathbb{P}^{t_{i}-u_{i}} \times \mathbb{P}^{t_{j}-u_{j}-1}\right)\right) \\
= & \left.\sum_{\left(u_{1}^{\prime}, \cdots, u_{n}^{\prime}\right) \in D(k+1)}\right) c_{\left(u_{1}^{\prime}, \cdots, u_{n}^{\prime}\right)}^{\prime} \Pi_{i=1}^{n} \mathbb{P}^{t_{i}-u_{i}^{\prime}},
\end{aligned}
$$

where all $c_{\left(u_{1}^{\prime}, \cdots, u_{n}^{\prime}\right)}^{\prime}=\sum_{\left(u_{1}, \cdots, u_{n}\right) \in \mathscr{M}\left(u_{1}^{\prime}, \cdots, u_{n}^{\prime}\right)} c_{\left(u_{1}, \cdots, u_{n}\right)} \cdot c_{n\left(u_{1}, \cdots, u_{n}\right)}$ with

$\mathscr{M}\left(u_{1}^{\prime}, \cdots, u_{n}^{\prime}\right)=\left\{\left(u_{1}, \cdots, u_{n}\right) \in D(k) ; \exists n\left(u_{1}, \cdots, u_{n}\right) \in \mathbb{Z}_{\geq 0}\right.$ with $\left.\left(u_{1}, \cdots, u_{n}\right)+e_{n\left(u_{1}, \cdots, u_{n}\right)}=\left(u_{1}^{\prime}, \cdots, u_{n}^{\prime}\right)\right\}$,

which clearly implies that $c_{\left(u_{1}^{\prime}, \cdots, u_{n}^{\prime}\right)}^{\prime}$ is positive for any $\left(u_{1}^{\prime}, \cdots, u_{n}^{\prime}\right) \in D(k+1)$.

Note that

$$
\left(\sum_{i=1}^{n} c_{i} \mathbb{P}^{t_{1}} \times \cdots \mathbb{P}^{t_{i}-1} \cdots \times \mathbb{P}^{t_{n}}\right)^{k}=f_{c}^{*} \mathbb{P}^{\ell-k}
$$

and then we have:

$$
X\left(V_{1}, \cdots, V_{n}\right) \cdot f_{c}^{*} \mathbb{P}^{\ell-k}=X\left(V_{1}, \cdots, V_{n}\right) \cdot \sum_{\left(u_{1}, \cdots, u_{n}\right) \in D(p)} c_{\left(u_{1}, \cdots, u_{n}\right)} \Pi_{i=1}^{n} \mathbb{P}^{m_{i}-u_{i}}
$$


Since $p$ is the largest integer with $M(p)=\left\{\left(m_{1}, \cdots, m_{n}\right): r+1-\sum_{i \in I}>d_{I}, \forall I \subset[n], \sum_{i=1}^{n} m_{i}=\right.$ $p\} \neq \emptyset$, we can take an element $\left(m_{1}, \cdots, m_{n}\right) \in M(p)$. By Proposition 3.1 for a general choice of $V^{i} \supset V_{i}$ satisfying the conditions in Proposition 3.1, $\Pi_{i=1}^{n} \mathbb{P}\left(V^{i} / V_{i}\right) \hookrightarrow \Pi_{i=1}^{n} \mathbb{P}\left(V / V_{i}\right)$ always has nonempty intersection with $X\left(V_{1}, \cdots, V_{n}\right)$. For $\left(u_{1}, \cdots, u_{n}\right) \in D(p) \backslash M(p)$, by Lemma 3.3. for a generic choice of $V^{i} \subset V$ with codim $V^{i}=j_{i}$, the closed subvariety $\Pi_{i=1}^{n} \mathbb{P}\left(V^{i} / V_{i}\right) \hookrightarrow \Pi_{i=1}^{n} \mathbb{P}\left(V / V_{i}\right)$ has empty intersection with $X\left(V_{1}, \cdots, V_{n}\right)$. Then we have:.

$$
\begin{aligned}
X\left(V_{1}, \cdots, V_{n}\right) \cdot f_{c}^{*} \mathbb{P}^{\ell-p} & =X\left(V_{1}, \cdots, V_{n}\right) \cdot \sum_{\left(u_{1}, \cdots, u_{n}\right) \in D(p)} c_{\left(u_{1}, \cdots, u_{n}\right)} \Pi_{i=1}^{n} \mathbb{P}^{t_{i}-u_{i}} \\
& =X\left(V_{1}, \cdots, V_{n}\right) \cdot \sum_{\left(u_{1}, \cdots, u_{n}\right) \in M(p)} c_{\left(u_{1}, \cdots, u_{n}\right)} \Pi_{i=1}^{n} \mathbb{P}^{t_{i}-u_{i}} \\
& =\sum_{\left(u_{1}, \cdots, u_{n}\right) \in M(p)} c_{\left(u_{1}, \cdots, u_{n}\right)}\left(\Pi_{i=1}^{n} \mathbb{P}_{t_{i}-u_{i}} \cdot X\left(V_{1}, \cdots, V_{n}\right)\right)
\end{aligned}
$$

Note that

$$
\begin{aligned}
& f_{c_{*}}\left(\sum_{\left(u_{1}, \cdots, u_{n}\right) \in M(p)} c_{\left(u_{1}, \cdots, u_{n}\right)}\left(\Pi_{i=1}^{n} \mathbb{P}^{m_{i}-u_{i}} \cdot X\left(V_{1}, \cdots, V_{n}\right)\right)\right) \\
= & \left(\sum_{\left(u_{1}, \cdots, u_{n}\right) \in M(p)} c_{\left(u_{1}, \cdots, u_{n}\right)} f_{c *}\left(\Pi_{i=1}^{n} \mathbb{P}^{t_{i}-u_{i}} \cdot X\left(V_{1}, \cdots, V_{n}\right)\right) .\right.
\end{aligned}
$$

By the projection formula, the intersection class $f_{c *}\left(X\left(V_{1}, \cdots, V_{n}\right)\right) \cdot \mathbb{P}^{\ell-p}$ is also $\left(\sum_{\left(u_{1}, \cdots, u_{n}\right) \in M(p)} c_{\left(u_{1}, \cdots, u_{n}\right)} f_{c *}\left(\Pi_{i=1}^{n} \mathbb{P}^{t_{i}-u_{i}} \cdot X\left(V_{1}, \cdots, V_{n}\right)\right)\right.$. The intersection product $\Pi_{i=1}^{n} \mathbb{P}^{t_{i}-u_{i}}$. $X\left(V_{1}, \cdots, V_{n}\right)$ corresponds to a nontrivial closed subvariety of $\Pi_{i=1}^{n} \mathbb{P}\left(V / V_{i}\right)$, which is in turn a closed subvariety of $\mathbb{P}^{\ell}$ under the closed immersion $f_{c}$. Hence $f_{c_{*}}\left(\Pi_{i=1}^{n} \mathbb{P}^{t_{i}-u_{i}} \cdot X\left(V_{1}, \cdots, V_{n}\right)\right)$ corresponds to a closed subvariety of $\mathbb{P}^{\ell}$ and $c_{\left(u_{1}, \cdots, u_{n}\right)}$ is a positive integer for each $\left(u_{1}, \cdots, u_{n}\right) \in M(p)$. Therefore, $\left(\sum_{\left(u_{1}, \cdots, u_{n}\right) \in M(p)} c_{\left(u_{1}, \cdots, u_{n}\right)} f_{c_{*}}\left(\Pi_{i=1}^{n} \mathbb{P}^{m_{i}-u_{i}} \cdot X\left(V_{1}, \cdots, V_{n}\right)\right)\right.$ is a nontrivial class in the Chow ring of $\mathbb{P}^{\ell}$, which implies that the codimension $p$ closed subvariety $\mathbb{P}^{\ell-p}$ always has nontrivial intersection with $f_{c_{*}}\left(X\left(V_{1}, \cdots, V_{n}\right)\right)$. Hence we have $r^{\prime} \geq p$, where $r^{\prime}$ is the dimension of $X\left(V_{1}, \cdots, V_{n}\right)$.

Note that $M(p+1)=\emptyset$, so for any $\left(j_{1}, \cdots, j_{n}\right)$ with $\sum_{i=1}^{n} j_{i}=p+1$, there exists $I \subset[n]$, with $r+1-\sum_{i \in I} j_{i} \leq d_{I}$, then by Lemma 3.3, for a generic choice of $V^{i} \subset V$ with $\operatorname{codim} V^{i}=j_{i}$, the closed subvariety $\Pi_{i=1}^{n} \mathbb{P}\left(V^{i} / V_{i}\right) \hookrightarrow \Pi_{i=1}^{n} \mathbb{P}\left(V / V_{i}\right)$ has empty intersection with $X\left(V_{1}, \cdots, V_{n}\right)$. Therefore we have:

$$
\begin{aligned}
X\left(V_{1}, \cdots, V_{n}\right) \cdot f_{c}^{*} \mathbb{P}^{\ell-(p+1)} & =X\left(V_{1}, \cdots, V_{n}\right) \cdot \sum_{\left(u_{1}, \cdots, u_{n}\right) \in D(p)} c_{\left(u_{1}, \cdots, u_{n}\right)} \Pi_{i=1}^{n} \mathbb{P}^{m_{i}-u_{i}} \\
& =0 .
\end{aligned}
$$

Therefore, $\left(f_{c *} X\left(V_{1}, \cdots, V_{n}\right)\right) \cdot \mathbb{P}^{\ell-k}$ is also trivial by the projection formula, which implies the codimension $p$ closed subvariety $\mathbb{P}^{\ell-(p+1)}$ always has empty intersection with $f_{c *}\left(X\left(V_{1}, \cdots, V_{n}\right)\right)$. Hence we have $r^{\prime}<(p+1)$. Combining with the fact $r^{\prime} \geq p$, we conclude

$$
r^{\prime}=\operatorname{dim}\left(X\left(V_{1}, \cdots, V_{n}\right)\right)=p
$$

In summary, we have proven the domain of the multi-degree function is $D(p)=\left\{\left(m_{1}, \cdots, m_{n}\right) \in\right.$ $\left.\mathbb{Z}_{\geq 0}^{n}: \sum_{i=1}^{n} m_{i}=p\right\}$. By Lemma $\underline{3.3}$, for any $\left(h_{1}, \cdots, h_{n}\right) \in D(p) \backslash M(p)$, there exists $V^{i} \supset V_{i}$ with 
$\operatorname{codim} V^{i}=h_{i}$, such that $\Pi_{i=1}^{n} \mathbb{P}\left(V^{i} / V_{i}\right) \hookrightarrow \Pi_{i=1}^{n} \mathbb{P}\left(V / V_{i}\right)$ has empty intersection with $X\left(V_{1}, \cdots, V_{n}\right)$, therefore multi-degree function takes zero at $D(p) \backslash M(p)$.

As for any $\left(m_{1}, \cdots, m_{n}\right) \in M(p)$, for any choice of $V^{i} \supset V_{i}$ with codim $V^{i}=m_{i}$, the closed subvariety $\Pi_{i=1}^{n} \mathbb{P}\left(V^{i} / V_{i}\right) \hookrightarrow \Pi_{i=1}^{n} \mathbb{P}\left(V / V_{i}\right)$ always has nonempty intersection with $X\left(V_{1}, \cdots, V_{n}\right)$, so we conclude that multi-degree function will take a nonzero value in $M(p)$.

Finally, we need to answer if multi-degree function takes a nonzero value at $\left(m_{1}, \cdots, m_{n}\right)$, then what is the nonzero value? Let's first recall an elementary lemma about vector bundles on $X / k$ where $X / k$ is a scheme over $k$.

Lemma 3.5. - Let $X / k$ be a scheme of finite type over $k$, and let

$$
0 \longrightarrow \mathscr{F}_{1} \stackrel{f}{\longrightarrow} \mathscr{F}_{2}
$$

be a short exact sequence of vector bundles on $X / k$. If Coker $f$ is also locally free and $\operatorname{Rank} \mathscr{F}_{1}=$ Rank $\mathscr{F}_{2}$, then $f$ is an isomorphism.

\section{Proof:}

It suffices to check this on the level of stalks. Let $p \in X / k$ be a point of $X / k$, notice that affine locally that exact sequence holds and localization is an exact functor, thus we have the following stalk locally at $p$ :

$$
0 \longrightarrow\left(\mathscr{F}_{1}\right)_{p} \stackrel{f_{p}}{\longrightarrow}\left(F_{2}\right)_{p} \longrightarrow(\operatorname{Coker} f)_{p} \longrightarrow 0
$$

Also we have $(\operatorname{Coker} f)_{p}$ is a free module over a local ring, thus is projective. Therefore we have a splitting, $\left(\mathscr{F}_{1}\right)_{p} \underset{f_{p}^{\prime}}{\stackrel{f_{p}}{\rightleftarrows}}\left(F_{2}\right)_{p}$ with $f_{p}^{\prime} \circ f_{p}=\mathrm{id}_{\left(\mathscr{F}_{1}\right)_{p}}$, thus $\left.\left.\mathscr{F}_{1}\right|_{p} \stackrel{\left.f\right|_{p}}{\longrightarrow} \mathscr{F}_{2}\right|_{p}$ is also an injection. Let's consider the following right exact sequence:

$$
\left.\left.\mathscr{F}_{1}\right|_{p} \stackrel{\left.f\right|_{p}}{\longrightarrow} \mathscr{F}_{2}\right|_{p} \longrightarrow \text { Coker }\left.f\right|_{p} \longrightarrow 0
$$

where $\left.f\right|_{p}$ is an injection. But we have Rank $\mathscr{F}_{1}=\operatorname{Rank} \mathscr{F}_{2}$, which implies $\operatorname{dim}\left(\left.\mathscr{F}_{1}\right|_{p}\right)=\operatorname{dim}\left(\left.\mathscr{F}_{2}\right|_{p}\right)$ as $k_{o}$ vector spaces, thus Coker $\left.f\right|_{p}=\{0\}$. By Nakayama's lemma, $(\operatorname{Coker} f)_{p}=0$ for any $p \in X / k$, so Coker $f=0$. Therefore we conclude that $f: \mathscr{F}_{1} \rightarrow \mathscr{F}_{2}$ is an isomorphism.

The following functor we are going to define represents the intersection of $X\left(V_{1}, \cdots, V_{n}\right)$ with $\Pi_{i=1}^{n} \mathbb{P}\left(V^{i} / V_{i}\right)$ in $\Pi_{i=1}^{n} \mathbb{P}\left(V / V_{i}\right)$, where $\left\{V^{i}\right\}_{i=1}^{n}$ were defined in Proposition 3.1 ,

Definition 3.6. - Let $\mathfrak{H}$ be a functor:

$$
(\mathbf{S c k} / \mathbf{k})^{\mathrm{op}} \rightarrow \text { Sets }
$$


and for each $S / k$, we associate the tuples of vector sub-bundles $\left(\mathscr{W}_{1}, \cdots, \mathscr{W}_{n}\right)$ of $V \otimes \mathscr{O}_{S}$ and $\operatorname{Rank}\left(\mathscr{W}_{i}\right)=\operatorname{dim} V_{i}+1$ for each $i \in[n]$ with the following properties:

(a) for any $I \subset[n]$ and $g_{I}:\left(V / \cap_{i \in I} V_{i}\right) \otimes \mathscr{O}_{S} \rightarrow \bigoplus_{i \in I}\left(V \otimes \mathscr{O}_{S}\right) / \mathscr{W}_{i}$, we have $\bigwedge^{r+1-d_{I}} g_{I}=0$

(b) $\mathscr{W}_{i}$ is a sub-bundle of $V^{i} \otimes \mathscr{O}_{S}$ for each $i \in[n]$.

(c) for each $i \in[n]$, we have $0 \rightarrow V_{i} \otimes \mathscr{O}_{S} \stackrel{f_{i}}{\longrightarrow} \mathscr{W}_{i} \stackrel{g_{i}}{\longrightarrow} \mathscr{V}$ where $f_{i}, g_{i}$ and $g_{i} \circ f_{i}$ all have locally free cokernels.

Proposition 3.7. - The functor $\mathfrak{H}$ is a one point functor and thus, the multi-degree is one.

\section{Proof:}

Recall at the end of the Proof of Proposition 3.1, we have $v_{[n]} \in \bigcap_{i=1}^{n} V^{i} \backslash \bigcup_{i=1}^{n} V_{i}$. It suffices to prove that $\mathscr{W}_{i}=\operatorname{Span}\left(V_{i}, v_{[n]}\right) \otimes \mathscr{O}_{S}$ for each $0 \leq i \leq n$ on any $S / k$. For the rest of the proof, let's fix a scheme $S / k$.

For any $i \in[n]$, define $J_{i}=\{I \subset[n]: i \in I\}$. For $\left(m_{1}, \cdots, m_{n}\right) \in M(p)$, set $k_{i}=\min \{(r+1-$ $\left.\left.\sum_{i \in I} m_{i}-d_{I}\right), \forall I \in J_{i}\right\}$. A priori, $k_{i} \geq 1$ and for each $i \in[n]$, we have $m_{i} \leq r-d_{i}$.

I claim that $k_{i}=1$. If not, consider $\left(m_{1}^{\prime}, \cdots, m_{i}^{\prime}, \cdots, m_{n}^{\prime}\right)$ where

$$
m_{p}^{\prime}=\left\{\begin{array}{cc}
m_{p}, & p \neq i \\
m_{p}+1, & p=i
\end{array}\right.
$$

$\left(m_{1}^{\prime}, \cdots, m_{n}^{\prime}\right)$ also satisfies for any $I \subset[n], r+1-\sum_{i \in I} m_{i}^{\prime}>d_{I}$, but $\sum_{i=1}^{n} m_{i}^{\prime}=p+1$, which contradicts that $p$ is the maximal number such that $M(p) \neq \emptyset$.

Therefore, $k_{i}=1$, then there exits $I_{i} \subset[n]$ where $i \in I_{i}$ such that $r+1-\sum_{k \in I_{i}} m_{k}=d_{I_{i}}+1$.

Since $\cap_{k \in I_{i}} V_{k} \otimes \mathscr{O}_{S}$ is a sub-bundle of $\mathscr{W}_{k}$ for each $k \in I_{i}$, and each $\mathscr{W}_{k}$ is a sub-bundle of $V^{k} \otimes \mathscr{O}_{S}$ then for each $k \in I_{i}$, the bundle morphism $\cap_{k \in I_{i}} V_{k} \otimes \mathscr{O}_{S} \rightarrow V^{k} \otimes \mathscr{O}_{S}$ factors through $\mathscr{W}_{k}$. Thus the natural morphism

$$
\left(V / \cap_{k \in I_{i}} V_{k}\right) \otimes \mathscr{O}_{S} \rightarrow \bigoplus_{k \in I_{i}}\left(V / V^{k}\right) \otimes \mathscr{O}_{S}
$$

factors through $\bigoplus_{k \in I_{i}}\left(\left(V \otimes \mathscr{O}_{S}\right) /\left(\mathscr{W}_{k}\right)\right)$. Therefore, we have the following natural morphism:

$$
\left(V / \cap_{k \in I_{i}} V_{k}\right) \otimes \mathscr{O}_{S} \stackrel{g_{I_{i}}}{\longrightarrow} \bigoplus_{k \in I_{i}}\left(\left(V \otimes \mathscr{O}_{S}\right) /\left(\mathscr{W}_{k}\right)\right) \stackrel{g_{w_{I_{i}}}}{\longrightarrow} \bigoplus_{i \in I_{i}}\left(V / V^{k}\right) \otimes \mathscr{O}_{S}
$$

Note that $\bigwedge^{r-d_{I_{i}}} g_{I_{i}}$ is a unit ideal sheaf $\left(\bigwedge^{r-d_{I_{i}}} g_{w_{I_{i}} \circ g_{I_{i}}}\right.$ is a unit ideal sheaf $)$ and by the definition of $\mathfrak{H}$, we have $\bigwedge^{r+1-d_{I_{i}}} g_{I_{i}}=0$. Then by Prop. 20.8 [2], $\operatorname{ker} g_{I_{i}}$ is locally free. Hence for each $p \in S / k$, we have

$$
\left.\left(\operatorname{ker} g_{I_{i}}\right)\right|_{p} \cong \operatorname{ker}\left(\left.g_{I_{i}}\right|_{p}\right)=\left(\left.\cap_{k \in I_{i}} \mathscr{W}_{k}\right|_{p}\right) /\left(\left.\cap_{k \in I_{i}} V_{k} \otimes \mathscr{O}_{S}\right|_{p}\right) .
$$

Notice that the natural morphism 
and $\operatorname{ker}\left(g_{w_{I_{i}}} \circ g_{I_{i}}\right)=\left(\left(\cap_{i \in I} V^{i}\right) /\left(\cap_{k \in I_{i}} V_{k}\right)\right) \otimes \mathscr{O}_{S}$, then there is a natural sheaf injection

$$
h_{I_{i}}: \quad \operatorname{ker} g_{I_{i}} \rightarrow \operatorname{ker}\left(g_{w_{I_{i}}} \circ g_{I_{i}}\right)=\left(\left(\cap_{k \in I_{i}} V^{k}\right) /\left(\cap_{k \in I_{i}} V_{k}\right)\right) \otimes \mathscr{O}_{S}
$$

$h_{I_{i}}$ is an injection of line bundles. Next I will prove that $h_{I_{i}}$ is an isomorphism. It suffices to show that Coker $h_{I_{i}}=0$. By a geometric Nakayama argument, it suffices to show that $\left.\left(\right.$ Coker $\left.h_{I_{i}}\right)\right|_{p}=0$ for each $p \in S / k$. Since there is a natural surjection (actually, isomorphism):

$$
\left.\left(\text { Coker }\left.h_{I_{i}}\right|_{p}\right) \rightarrow\left(\operatorname{Coker} h_{I_{i}}\right)\right|_{p} \rightarrow 0,
$$

it suffices to show Coker $\left.h_{I_{i}}\right|_{p}=0$.

Fiber-wise at $p \in S / k$, for $\left.g_{I_{i}}\right|_{p}: V /\left.\left.\cap_{k \in I_{i}} V_{k} \otimes \mathscr{O}_{S}\right|_{p} \rightarrow\left(\bigoplus_{k \in I_{i}} \mathscr{V}_{S} / \mathscr{W}_{k}\right)\right|_{p}$, we have $\bigwedge^{r+1-d_{I_{i}}}=0$, this implies $\left.\left.\cap_{k \in I_{i}} V_{k} \otimes \mathscr{O}_{S}\right|_{p} \varsubsetneqq \cap_{k \in I_{i}} \mathscr{W}_{k}\right|_{p}$, which implies that $d_{I_{i}}<\operatorname{dim}\left(\left.\cap_{k \in I_{i}} \mathscr{W}_{k}\right|_{p}\right)$. A priori, $\left.\left.\cap_{k \in I_{i}} \mathscr{W}_{k}\right|_{p} \subset \cap_{k \in I_{i}} V^{k} \otimes \mathscr{O}_{S}\right|_{p}$, which implies $\operatorname{dim}\left(\left.\cap_{k \in I_{i}} \mathscr{W}_{k}\right|_{p}\right) \leq \operatorname{dim}\left(\left.\cap_{k \in I_{i}} V^{k} \otimes \mathscr{O}_{S}\right|_{p}\right)=d_{I}+1$. Therefore, $\operatorname{dim}\left(\left.\cap_{k \in I_{i}} \mathscr{W}_{k}\right|_{p}\right)=\operatorname{dim}\left(\left.\cap_{k \in I_{i}} V^{k} \otimes \mathscr{O}_{S}\right|_{p}\right)$, so we conclude that $\left.\cap_{k \in I_{i}} \mathscr{W}_{k}\right|_{p}=\left.\cap_{k \in I_{i}} V^{i} \otimes \mathscr{O}_{S}\right|_{p}$, and therefore

$$
\left(\left.\cap_{k \in I_{i}} \mathscr{W}_{k}\right|_{p}\right) /\left(\left.\cap_{k \in I_{i}} V_{k} \otimes \mathscr{O}_{S}\right|_{p}\right)=\left.\left(\left(\cap_{k \in I_{i}} V^{k}\right) /\left(\cap_{k \in I_{i}} V_{k}\right)\right) \otimes \mathscr{O}_{S}\right|_{p}
$$

Hence we have that Coker $\left.h_{I_{i}}\right|_{p}=0$, thus $\left.\left(\operatorname{Coker} h_{I_{i}}\right)\right|_{p}=0$ for any $p \in S / k$, therefore we conclude that $h_{I_{i}}$ is an isomorphism of line bundles. Equivalently, we have:

$$
\operatorname{ker} g_{I_{i}}=\operatorname{ker}\left(g_{w_{I_{i}}} \circ g_{I_{i}}\right)=\left(\left(\cap_{k \in I_{i}} V^{k}\right) /\left(\cap_{k \in I_{i}} V_{k}\right)\right) \otimes \mathscr{O}_{S}
$$

Since $\operatorname{ker} g_{I_{i}}=\left(\left(\cap_{k \in I_{i}} V^{k}\right) /\left(\cap_{k \in I_{i}} V_{k}\right)\right) \otimes \mathscr{O}_{S}$ and $\mathscr{W}_{k}$ contains $\left(\cap_{k \in I_{i}} V^{k}\right) \otimes \mathscr{O}_{S}$ as a sub-bundle for each $k \in I_{i}$, we have $\left(\cap_{k \in I_{i}} V^{k}\right) \otimes \mathscr{O}_{S}$ is the kernel of $\mathscr{V}_{S} \rightarrow \oplus_{k \in I_{i}} \mathscr{V}_{S} / \mathscr{W}_{k}$, thus for each $k \in I_{i}$, the zero morphism $\left(\cap_{k \in I_{i}} V^{k}\right) \otimes \mathscr{O}_{S} \hookrightarrow \mathscr{V}_{S} \rightarrow \mathscr{V}_{S} / \mathscr{W}_{k}$ will factor through $\mathscr{W}_{k}$, which is still a sub-vector bundle of $\mathscr{V}_{S}$. Therefore, $\cap_{k \in I_{i}} V^{k} \otimes \mathscr{O}_{S}$ is a sub-bundle of $\mathscr{W}_{k}$.

Recall that $v_{[n]} \in\left(\bigcap_{i=1}^{n} V^{i}\right) \backslash\left(\bigcup_{i=1}^{n} V_{i}\right)$, thus $v_{[n]} \in \bigcap_{k \in I_{i}} V^{k}$. Hence $\operatorname{Span}\left(v_{[n]}\right) \otimes \mathscr{O}_{S}$ is a sub-bundle of $\mathscr{W}_{i}$. Then we have $\operatorname{Span}\left(V_{i}, v_{[n]}\right) \otimes \mathscr{O}_{S}$ is a sub-bundle of $\mathscr{W}_{i}$, but notice that

$$
\operatorname{Rank}\left(\operatorname{Span}\left(V_{i}, v_{[n]}\right) \otimes \mathscr{O}_{S}\right)=d_{i}+1=\operatorname{Rank} \mathscr{W}_{i}
$$

by $\operatorname{Lemma} 3.5, \operatorname{Span}\left(V_{i}, v_{[n]}\right) \otimes \mathscr{O}_{S}=\mathscr{W}_{i}$.

By Case (i) and (ii), $\mathscr{W}_{i}=\operatorname{Span}\left(V_{i}, v_{[n]}\right) \otimes \mathscr{O}_{S}$ for each $1 \leq i \leq n$. We conclude that for each scheme $S / k$, the set $\mathfrak{H}(S)=\left\{\left(\operatorname{Span}\left(V_{1}, v_{[n]}\right) \otimes \mathscr{O}_{S}, \cdots, \operatorname{Span}\left(V_{n}, v_{[n]}\right) \otimes \mathscr{O}_{S}\right)\right\}$, a one-point functor.

Corollary 3.8. - The moduli functor $\mathfrak{F}_{1}$ is multiplicity-free and hence Cohen-Macaulay.

\section{Proof:}

By Proposition 3.1 and Lemma 3.3 , whenever $\sum_{i \in[n]} c_{i}=p$, we an find $\left\{V^{i} \subset V\right\}_{i=1}^{n}$, such that $\Pi_{i=1}^{n} \mathbb{P}\left(V^{i} / V_{i}\right)$ intersects with $\mathfrak{F}_{1}$ at most one point, and by Proposition 3.7 , if it intersects at one point, 
the multiplicity will be one. Therefore we conclude the closure of the image of the rational map $\mathbb{P}(V) \rightarrow \Pi_{i=1}^{n} \mathbb{P}\left(V / V_{i}\right)$ is multiplicity-free, then by a result of Brion in [1], it is Cohen-Macaulay.

\section{INITIAL DEgEnERATION OF $X\left(V_{1}, \cdots, V_{n}\right)$}

In this section, we will study the initial degeneration of $X\left(V_{1}, \cdots, V_{n}\right)$ under a prescribed term order.

\subsection{Genericity Setup}

Let $\left\{V_{i}\right\}_{i=1}^{n}$ be a finite sequence of subspaces of $V$, and denote $d_{I}=\operatorname{dim}\left(\cap_{i \in I} V_{i}\right)$.

Consider the natural map $V \rightarrow \Pi_{i=1}^{n} V / V_{i}$. Choose coordinate system for $V$ and $\left\{V / V_{i}\right\}_{i=1}^{n}$ in such a generic way that for $A_{i}: V \rightarrow V / V_{i}$, following matrix

$$
A_{I}=\left[\begin{array}{c}
A_{1} \\
\vdots \\
A_{n}
\end{array}\right]
$$

has the following property:

Let $C$ be any set of row vectors in $A_{I}$, which takes $a_{m}$ rows taken in $A_{m}$. Let $I_{C}$ to be the set $\left\{i \mid i \in[n], C \cap\left\{\right.\right.$ row vectors in $\left.\left.A_{i}\right\} \neq \emptyset\right\}$. If for any $I^{\prime} \subset I_{C}, \sum_{m \in I^{\prime}} a_{m} \leq r+1-d_{I^{\prime}}$ ( we will call this condition as Condition $(\star))$, then $\operatorname{dim}(\operatorname{Span} C)=|C|$.

Remark 4.1. - Such a way of choosing basis to satisfy the genericity set-up always exists. A priori, for arbitrary choice of basis of $V$ and $V / V_{i}$ 's whose corresponding matrix is $A_{I}^{\prime}$, there exists one set $C$ of row vectors with condition $(\star)$ has $\operatorname{dim}(\operatorname{Span} C)=|C|$, and row operations which keep this property for $C$ form a dense open subset of $\Pi_{i=1}^{n} G l\left(V / V_{i}\right)$ and denote this open subset as $U_{C}$. For another set $C^{\prime}$ with condition $(\star)$ and $\left|C^{\prime}\right|=|C|$, we can do elementary row operation to permute the rows between $C^{\prime}$ and $C$ so that $\operatorname{dim}\left(\operatorname{Span} C^{\prime}\right)=\left|C^{\prime}\right|$, thus there is a nonempty dense open row operations in $\Pi_{i=1}^{n} G l\left(V / V_{i}\right)$ which makes $\operatorname{dim}\left(\operatorname{Span} C^{\prime}\right)=\left|C^{\prime}\right|$ and we will denote this subset as $U_{C^{\prime}}$. There are only finitely many $C^{\prime}$ with condition $(\star)$ in the collection of subsets of the set of row vectors and for each $C^{\prime}$.

Let $I_{\star}=$ row vectors of $\mathrm{A}_{\mathrm{I}}$ with Property $\left.(\star)\right\}$, and it is clear that $I_{\star}$ is finite. $\bigcap_{C^{\prime} \in I_{\star}} U_{C^{\prime}}$ is nonempty because each $U_{C^{\prime}}$ is nonempty and dense open. Take $\beta \in \bigcap_{C^{\prime} \in I_{\star}} U_{C^{\prime}}$, then $\beta \cdot A_{I}^{\prime}$ will satisfy the genericity setup. 


\subsection{Prime Decomposition of the Initial Ideal}

Recall that $M(h)=\left\{\left(m_{1}, \cdots, m_{n}\right) \in \mathbb{Z}_{\geq 0}^{n}: \forall I \subset[n], r+1-\sum_{i \in I} m_{i}>d_{I}\right\}$ and $M(\operatorname{dim}(\overline{\operatorname{Im} f}))=$ $M(p)$, where $p=\max \{h: M(h) \neq \emptyset\}$.

Definition 4.1. - $\widehat{M}=\left\{\left(m_{1}, \cdots, m_{n}\right) \in \bigcup_{h=1}^{p} M(h), \forall k \in[n], \exists I_{k} \subset[n], k \in I_{k}, r+1-\sum_{i \in I_{k}} m_{i}=d_{I_{k}}+\right.$ $1\}$.

A priori, $M(p) \subset \widehat{M}$. Since $d_{I}$ can be realised as the dimension of intersection of linear subspaces of a vector space, $M(p)$ and $\widehat{M}$ are actually equal, which is proved in the following proposition.

Proposition 4.2. $-M(p)=\widehat{M}$

Proof: Given an $\left(m_{1}, \cdots, m_{n}\right) \in \widehat{M}$, for each $1 \leq h \leq n$, let $S_{m_{h}}:=\left\{I \subset[n]: h \in I, r+1-\sum_{i \in I} m_{i}=\right.$ $\left.d_{I}+1\right\}$, which is non empty by the definition of $\widehat{M}$ and forms a partially ordered set under inclusion. I claim that $S_{m_{h}}$ has a unique maximal element.

Suppose that $I_{1}$ and $I_{2}$ are both maximal. Consider the intersection $I_{1} \cap I_{2}$ which still contains $h$.

$$
\begin{aligned}
r+1-\sum_{i \in I_{1} \cup I_{2}} m_{i} & =\left(r+1-\sum_{i \in I_{1}} m_{i}\right)+\left(r+1-\sum_{i \in I_{2}} m_{i}\right)-\left(r+1-\sum_{i \in\left(I_{1} \cap I_{2}\right)} m_{i}\right) \\
& =\left(d_{I_{1}}+1\right)+\left(d_{I_{2}}+1\right)-\left(r+1-\sum_{i \in\left(I_{1} \cap I_{2}\right)} m_{i}\right) .
\end{aligned}
$$

On the other hand, $d_{I_{1}}=\operatorname{dim}\left(\bigcap_{i \in I_{1}} V_{i}\right)$ and $d_{I_{2}}=\operatorname{dim}\left(\bigcap_{i \in I_{2}} V_{i}\right)$, and both $\bigcap_{i \in I_{1}} V_{i}$ and $\bigcap_{i \in I_{2}} V_{i}$ are linear subspaces of $\bigcap_{i \in\left(I_{1} \cap I_{2}\right)} V_{i}$, thus

$$
\begin{aligned}
\operatorname{Span}\left(\cap_{i \in I_{1}} V_{i}, \cap_{i \in I_{2}} V_{i}\right) \subset \bigcap_{i \in\left(I_{1} \cap I_{2}\right)} V_{i} . \\
\left.d_{I_{1}+d_{I_{2}}-d_{I_{1} \cup I_{2}}}=\operatorname{dim}\left(\cap_{i \in I_{1}} V_{i}\right)+\operatorname{dim}\left(\cap_{i \in I_{2}} V_{i}\right)\right)-\operatorname{dim}\left(\cap_{i \in I_{1} \cup I_{2}} V_{i}\right) \\
=\operatorname{dim} \operatorname{Span}\left(\cap_{i \in I_{1}} V_{i}, \cap_{i \in I_{2}} V_{i}\right) \\
\leq \operatorname{dim}\left(\cap_{i \in\left(I_{1} \cap I_{2}\right)} V_{i}\right)=d_{I_{1} \cap I_{2}} \\
<\left(r+1-\sum_{i \in\left(I_{1} \cap I_{2}\right)} m_{i}\right) .
\end{aligned}
$$

Therefore we have

$$
\begin{aligned}
r+1-\sum_{i \in I_{1} \cup I_{2}} m_{i} & =\left(d_{I_{1}}+1\right)+\left(d_{I_{2}}+1\right)-\left(r+1-\sum_{i \in\left(I_{1} \cap I_{2}\right)} m_{i}\right) \\
& =\left(d_{I_{1}}+d_{I_{2}}-d_{I_{1} \cup I_{2}}\right)+2+d_{I_{1} \cup I_{2}}-\left(r+1-\sum_{i \in\left(I_{1} \cap I_{2}\right)} m_{i}\right) \\
& <\left(r+1-\sum_{i \in\left(I_{1} \cap I_{2}\right)} m_{i}\right)+2+d_{I_{1} \cup I_{2}}-\left(r+1-\sum_{i \in\left(I_{1} \cap I_{2}\right)} m_{i}\right) \\
& =2+d_{I_{1} \cup I_{2}} .
\end{aligned}
$$

Note that $r+1-\sum_{i \in I_{1} \cup I_{2}} m_{i}>d_{I_{1} \cup I_{2}}$, and $r+1-\sum_{i \in I_{1} \cup I_{2}} m_{i}$ is an integer, $r+1-\sum_{i \in I_{1} \cup I_{2}} m_{i}$ has to be equal to $d_{I_{1} \cup I_{2}}+1$, therefore, $I_{1} \cup I_{2} \in S_{m_{h}}$, thus $I_{1} \subset I_{1} \cup I_{2}$, and $I_{2} \subset I_{1} \cup I_{2}$, but $I_{1}$ and $I_{2}$ are maximal, then $I_{1}=I_{1} \cup I_{2}=I_{2}$. Therefore $S_{m_{h}}$ has a unique maximal element.

Given $\left(m_{1}, \cdots, m_{n}\right) \in \widehat{M}$, and let $I_{m_{h}}$ be the unique maximal element of $S_{m_{h}}$, then I claim (i): $I_{m_{h}}=$ $I_{m_{j}}$ for any $j \in I_{m_{h}}$; (ii): $I_{m_{h}} \cap I_{m_{j}}=\emptyset$ for $j \notin I_{m_{h}}$. 
Proof of Claim (i): For any $j \in I_{m_{h}}$, we have $I_{m_{h}} \in S_{m_{j}}$, and since $I_{m_{j}}$ is the maximal element in $S_{m_{j}}$, we have $I_{m_{h}} \subset I_{m_{j}}$. Therefore, $h \in I_{m_{j}}$, then $I_{m_{j}} \in S_{m_{h}}$ and since $I_{m_{h}}$ is the maximal in $S_{m_{h}}$, we have $I_{m_{j}} \subset I_{m_{h}}$. We conclude that $I_{m_{h}}=I_{m_{j}}$.

Proof of Claim (ii): For any $j \notin I_{m_{h}}$, consider $I_{m_{j}}$, the maximal element of $S_{m_{j}}$. If $I_{m_{j}} \cap I_{m_{h}} \neq \emptyset$, take $q \in I_{m_{j}} \cap I_{m_{h}}$, then by claim one, $I_{m_{h}}=I_{m_{q}}=I_{m_{j}} \ni j$, thus $j \in I_{m_{h}}$, a contradiction. Therefore $I_{m_{h}} \cap I_{m_{j}}=\emptyset$ for $j \notin I_{m_{h}}$.

By Claim (i) and (ii),given any $\left(m_{1}, \cdots, m_{n}\right) \in \widehat{M}$, one can decompose $[n]$ into disjoint union $I_{u_{1}} \sqcup$ $I_{u_{2}} \cdots \sqcup I_{u_{\ell}}$ where $\left\{u_{1}, \cdots, u_{\ell}\right\} \subset[n]$, such that $r+1-\sum_{i \in I_{u_{q}}} m_{i}=d_{I_{u_{q}}}+1$ for each $1 \leq q \leq \ell$.

For each $\left(a_{1}, \cdots, a_{n}\right) \in M(p)$, we have $r+1-\sum_{i \in I_{u_{q}}} a_{i}>d_{I_{u_{q}}}$ but $r+1-\sum_{i \in I_{u_{q}}} m_{i}=d_{I_{u_{q}}}+1$, thus $\sum_{i \in I_{u_{q}}} a_{i} \leq \sum_{i \in I_{u_{q}}} m_{i}$ for each $1 \leq q \leq \ell$.

$$
\begin{aligned}
p=\sum_{i=1}^{n} a_{i} & =\sum_{q=1}^{\ell} \sum_{i \in I_{u q}} a_{i} \\
& \leq \sum_{q=1}^{\ell} \sum_{i \in I_{u q}} m_{i} \\
& =\sum_{i=1}^{n} m_{i}
\end{aligned}
$$

Recall that by definition, $\widehat{M} \subset \bigcup_{h=1}^{p} M(h)$, and therefore $\sum_{i=1}^{n} m_{i} \leq p$, thus $\sum_{i=1}^{n} m_{i}=p$. Hence for each $\left(m_{1}, \cdots, m_{n}\right) \in \widehat{M}$, we have $\sum_{i=1}^{n} m_{i}=p$, then $\widehat{M} \subset M(p)$. Thus the proposition follows.

Remark 4.2. - A priori, $M(p) \subset \widehat{M}$, and in general, this containment is strict. The proof of Proposition 4.2 assumes that $d_{I}$ can be realized as the dimension of intersections of linear subspaces of a single vector space, but actually, by exactly the same argument, one can prove the same result for general matroid rank functions.

Convention 4.3. - Recall $d_{i}=\operatorname{dim} V_{i}$. Let $k\left[x_{i, 1}, x_{i, 2}, \cdots, x_{i, r+1-d_{i}}\right]$ denote the projective coordinate ring of $\mathbb{P}\left(V / V_{i}\right)$, and our lex order is taken to be

$$
\begin{cases}x_{i, j} \succ x_{\ell, m} & \text { if } i>\ell \\ x_{i, j} \succ x_{\ell, m} & \text { if } i=\ell, j>m\end{cases}
$$

Definition 4.3. $-P_{\left(m_{1}, \cdots, m_{n}\right)}$ is a prime ideal associated to $\left(m_{1}, \cdots, m_{n}\right)$ defined as the prime ideal

$$
<x_{1,1}, x_{1,2}, \cdots, x_{1, r-d_{1}-m_{1}}, \ldots, x_{i, 1}, \cdots, x_{i, r-d_{i}-m_{i}}, \ldots, x_{n, 1}, \cdots, x_{n, r-d_{n}-m_{n}}>
$$

Definition 4.4. - Define $I_{o}$ to be $\bigcap_{\left(m_{1}, \cdots, m_{n}\right) \in M(p)} P_{\left(m_{1}, \cdots, m_{2}\right)}$.

Let's first make a explicit description of $I_{o}$ by writing down its generators.

Convention 4.4. - For a monomial $x_{\ell, \ell_{1}} x_{2, \ell_{2}} \cdots x_{i, \ell_{i}} \cdots x_{n, \ell_{n}}$ in the coordinate ring of $\Pi_{1}^{n} \mathbb{P}\left(V / V_{i}\right)$, when $\ell_{i}=0$, we set up $x_{i, \ell_{i}}=1$.

The following proposition writes down a set of generators of $I_{o}$. 
Proposition 4.5. - $I_{O}$ can be generated by monomials $x_{1, \ell_{1}} x_{2, \ell_{2}} \cdots x_{i, \ell_{i}} \cdots x_{n, \ell_{n}}$ where $\ell_{i}$ 's are non negative integers satisfying the following condition:

$$
\exists I \subset[n] \text { such that } r+1-\sum_{i \in I}\left(r+1-d_{i}-\ell_{i}\right) \leq d_{I}
$$

Before proving this proposition, let's first prove the following lemma:

Lemma 4.6. - Given a monomial $x_{1, \ell_{1}} x_{2, \ell_{2}} \cdots x_{i, \ell_{i}} \cdots x_{n, \ell_{n}}$, such that for any $I \in[n]$, we have $r+1-$ $\sum_{i \in I}\left(r+1-d_{i}-\ell_{i}\right)>d_{I}$, then this monomial is not in $I_{o}$.

Proof: For any $I \subset[n]$, we have $r+1-\sum_{i \in I}\left(r+1-d_{i}-\ell_{i}\right)>d_{I}$, therefore $\left(r+1-d_{1}-\ell_{1}, \cdots, r+\right.$ $\left.1-d_{n}-\ell_{n}\right) \in M(n)$ for some $n \leq p$. Hence there exists $\left(m_{1}, \cdots, m_{n}\right) \in \widehat{M}$ such that for each $1 \leq i \leq n$, we have $r+1-d_{i}-\ell_{i} \leq m_{i}$, which implies $r-d_{i}-m_{i}<\ell_{i}$ for each $i \in[n]$.

Consider the prime ideal $P_{\left(m_{1}, \cdots, m_{n}\right)}$, and we conclude that this monomial $x_{1, \ell_{1}} x_{2, \ell_{2}} \cdots x_{i, \ell_{i}} \cdots x_{n, \ell_{n}}$ is not in this prime ideal $P_{\left(m_{1}, \cdots, m_{n}\right)}$ because $x_{i, \ell_{i}} \notin P_{\left(m_{1}, \cdots, m_{n}\right)}$ for all $i$ 's.

By Lemma 4.2 $\widehat{M}=M(p)$, thus $\left(m_{1}, \cdots, m_{n}\right) \in M(p)$. The prime ideal $P_{\left(m_{1}, \cdots, m_{n}\right)}$ is a prime factor of $I_{o}$, therefore $x_{1, \ell_{1}} x_{2, \ell_{2}} \cdots x_{i, \ell_{i}} \cdots x_{n, \ell_{n}}$ is not in $I_{o}$, which is contained in $P_{\left(m_{1}, \cdots, m_{n}\right)}$.

Lemma 4.7. - Given a monomial $x_{1, \ell_{1}} x_{2, \ell_{2}} \cdots x_{i, \ell_{i}} \cdots x_{n, \ell_{n}}$, with an $I \subset[n]$ such that

$$
r+1-\sum_{i \in I}\left(r+1-d_{i}-\ell_{i}\right) \leq d_{I}
$$

then this monomial $x_{1, \ell_{1}} x_{2, \ell_{2}} \cdots x_{i, \ell_{i}} \cdots x_{n, \ell_{n}}$ is in $I_{o}$.

Proof: For any $\left(m_{1}, \cdots, m_{n}\right) \in M(p)$, we have for the $I \in[n]$ in this lemma, $r+1-\sum_{i \in I} m_{i}>d_{I}$. We also have $r+1-\sum_{i \in I}\left(r+1-d_{i}-\ell_{i}\right) \leq d_{I}$, so there exists $i^{\prime} \in I$, such that $m_{i^{\prime}}<r+1-d_{i^{\prime}}-$ $\ell_{i^{\prime}}$, thus $\ell_{i^{\prime}} \leq r-d_{i^{\prime}}-m_{i^{\prime}}$. Therefore, $x_{i^{\prime}, \ell_{i^{\prime}}} \in P_{\left(m_{1}, \cdots, m_{n}\right)}$, which implies $x_{1, \ell_{1}} x_{2, \ell_{2}} \cdots x_{i, \ell_{i}} \cdots x_{n, \ell_{n}} \in$ $P_{\left(m_{1}, \cdots, m_{n}\right)}$. Since $\left(m_{1}, \cdots, m_{n}\right)$ is an arbitrary element in $M(p)$, thus we have $x_{1, \ell_{1}} x_{2, \ell_{2}} \cdots x_{i, \ell_{i}} \cdots x_{n, \ell_{n}} \in$ $\cap_{\left(m_{1}, \cdots, m_{n}\right) \in M(p)} P_{\left(m_{1}, \cdots, m_{n}\right)}$

Lemma 4.8. - Generaters of $I_{o}$ can be written as monomials of the form $x_{1, \ell_{1}} x_{2, \ell_{2}} \cdots x_{i, \ell_{i}} \cdots x_{n, \ell_{n}}$

Proof: Intersections of monomial ideals are still monomial, thus $I_{o}$ is a monomial ideal. Let $I_{k}$ be a subset of $\left\{0,1,2, \cdots, r+1-d_{i}\right\}$ and $i_{k}$ be the smallest integer of $I_{k}$. If $\Pi_{i=1}^{n} \Pi_{h \in I_{k}} x_{i, h} \in I_{o}$, then $\Pi_{k=1}^{n} x_{k, i_{k}}$ is also in $I_{o}$.

Now let's prove the Proposition 4.5 .

Proof: By lemma4.8, we know that $I_{o}$ is generated by monomials of the form $x_{1, \ell_{1}} x_{2, \ell_{2}} \cdots x_{i, \ell_{i}} \cdots x_{n, \ell_{n}}$. And by Lemma4.6 and Lemma 4.7, we know that generators of $I_{o}$ are of the form $x_{1, \ell_{1}} x_{2, \ell_{2}} \cdots x_{i, \ell_{i}} \cdots x_{n, \ell_{n}}$ with $r+1-\sum_{i \in I}\left(r+1-d_{i}-\ell_{i}\right) \leq d_{I}$ for some $I \in[n]$. 
Definition 4.9. - A generator of the form $x_{1, \ell_{1}} x_{2, \ell_{2}} \cdots x_{i, \ell_{i}} \cdots x_{n, \ell_{n}}$ for $I_{o}$ is called a irredundant generator if $I=\left\{\ell_{i} \mid \ell_{i} \neq 0\right\}$ satisfies the condition that $r+1-\sum_{i \in I}\left(r+1-d_{i}-\ell_{i}\right) \leq d_{I}$ and for any $I^{\prime} \varsubsetneqq I$, $r+1-\sum_{i \in I^{\prime}}\left(r+1-d_{i}-\ell_{i}\right)>d_{I^{\prime}}$

Lemma 4.10. - Irredundant generators exist and all of the irredundant generators generate $I_{0}$.

Proof: For any $x_{1, \ell_{1}} x_{2, \ell_{2}} \cdots x_{i, \ell_{i}} \cdots x_{n, \ell_{n}} \in I_{o}$, there is $I_{1} \subset[n]$, with $r+1-\sum_{i \in I_{1}}\left(r+1-d_{i}-\ell_{i}\right) \leq d_{I_{1}}$, then $\Pi_{i \in I_{1}} x_{i, \ell_{i}} \in I_{o}$. Take $I_{2} \varsubsetneqq I_{1}$ with $r+1-\sum_{i \in I_{2}}\left(r+1-d_{i}-\ell_{i}\right) \leq d_{I_{2}}$, we can truncate this generator to the form $\Pi_{i \in I_{2}} x_{i, \ell_{i}}$. Repeating this procedure, we will get :

$$
I_{1} \supsetneqq I_{2} \cdots \supsetneqq I_{h} \cdots
$$

The sequence will stop eventually, and denote the last term in the sequence as $I_{k}$ then $\Pi_{i \in I_{k}} x_{i, \ell_{i}}$ is an irredundant generator. The same operation will reduce very monomial generators of the form $\Pi_{i=1}^{n} x_{i, \ell_{i}}$ into an irredundant generator.

In computer vision $[\mathbf{6}]$, the length of the generators (their generators corresponds to "irredundant generators" in our language) was bounded by 4 in Theorem 2.1. The next lemma gives a generalisation of this upper bound.

Corollary 4.11. - The length of the irredundant generators of $I_{o}$ is bounded by

$$
\min \{r+1, n\}
$$

Proof: By Lemma4.10, generators of the $I_{o}$ are monomials $x_{1, \ell_{1}} x_{2, \ell_{2}} \cdots x_{i, \ell_{i}} \cdots x_{n, \ell_{n}}$ with $\exists I \in[n]$ such that $r+1-\sum_{i \in I}\left(r+1-d_{i}-l_{i}\right) \leq d_{I}$ and for any $I^{\prime} \varsubsetneqq I, r+1-\sum_{i \in I^{\prime}}\left(r+1-d_{i}-\ell_{i}\right)>d_{I^{\prime}}$.

Pick $I^{\prime} \subset I$ with $\left|I^{\prime}\right|=|I|-1$. By $r+1-\sum_{i \in I^{\prime}}\left(r+1-d_{i}-\ell_{i}\right)>d_{I^{\prime}}$ and $\left(r+1-d_{i}-\ell_{i}\right) \geq 1$ for each $i,\left|I^{\prime}\right| \leq \sum_{i \in I^{\prime}}\left(r+1-d_{i}-\ell_{i}\right)<r+1-d_{I^{\prime}} \leq r+1$, therefore $|I|=\left|I^{\prime}\right|+1 \leq r+1$. On the other hand, $|I| \leq n$, thus this corollary follows.

Let $A_{i}$ be the matrix representation of the linear map $V \rightarrow V / V_{i}$, and $q_{i}$ be $\left(x_{i, 1}, \cdots, x_{i, r+1-d_{i}}\right)^{T}$, and let $I=\left\{\delta_{1}, \cdots, \delta_{|I|}\right\}$ be any subset $[n]$, and consider the following matrix associated to $I \subset[n]$,

$$
\left[\begin{array}{ccccc}
A_{\delta_{1}} & q_{\delta_{1}} & 0 & \ldots & 0 \\
A_{\delta_{2}} & 0 & q_{\delta_{2}} & \vdots & 0 \\
\vdots & \vdots & & & \\
A_{\delta_{|I|}} & 0 & \ldots & \ldots & q_{\delta_{|I|}}
\end{array}\right]
$$

Consider all of the $r+1-d_{I}+|I|$-minor of the above matrix, and denote the ideal generated by these minors as $I_{f}$.

Lemma 4.12. $-I_{o} \subset \operatorname{in}_{\prec} I_{f}$

Proof: It suffices to check that all of the irredundant generators of $I_{o}$ are in $\operatorname{in}_{\prec} I_{f}$. We are going to check monomials of the form $x_{1, \ell_{1}} \cdots x_{n, \ell_{n}}$ whose $I=\left\{\ell_{i}: \ell_{i} \neq 0\right\}$ has the property that $r+1-\sum_{i \in I}\left(r+1-d_{i}-\ell_{i}\right) \leq d_{I}$ and for any $I^{\prime} \varsubsetneqq I, r+1-\sum_{i \in I^{\prime}}\left(r+1-d_{i}-\ell_{i}\right)>d_{I^{\prime}}$. Now let 
$I=\left\{y_{1}, \cdots, y_{|I|}\right\}$, then we need to prove that $x_{y_{1}, \ell_{y_{1}}} \cdots x_{y_{|I|}, \ell_{|I|}} \in \mathrm{in}_{\prec} I_{f}$. Let us to consider matrix with the following form:

Denote $A_{i, j}$ be the $j$-th row of $A_{i}$; and let $I=\left\{y_{1}, \cdots, y_{|I|}\right\}$

$$
A^{\prime}=\left[\begin{array}{ccccc}
A_{y_{1}, l_{y_{1}}} & x_{y_{1}, \ell_{y_{1}}} & 0 & & \\
\vdots & \vdots & \vdots & 0 & \\
A_{y_{1}, r+1-d_{y_{1}}} & x_{y_{1}, r+1-d_{y_{1}}} & 0 & & \\
A_{y_{k}, l_{y_{k}}} & 0 & \ldots & x_{y_{k}, l_{y_{k}}} & 0 \\
\vdots & \vdots & \vdots & \vdots & \\
A_{y_{k}, r+1-d_{y_{k}}} & 0 & \ldots & x_{y_{k}, r+1-d_{y_{k}}} & 0 \\
A_{y_{|I|}, l_{|| I \mid}} & 0 & \ldots & 0 & x_{y_{|I|}, \ell_{|| I \mid}} \\
\vdots & \vdots & \vdots & 0 & \\
A_{y_{|I|}, r+1-d_{y_{|I|}}} & 0 & \ldots & 0 & x_{y_{|I|}, r+1-d_{y_{|I|}}}
\end{array}\right]
$$

In the matrix above, there are $\sum_{i \in I}\left(r+2-d_{i}-l_{i}\right)$ rows and $(r+1+|I|)$ columns, and by

$$
r+1-d_{I} \leq \sum_{i \in I}\left(r+1-d_{i}-l_{i}\right),
$$

we have

$$
\left(r+1-d_{I}+|I|\right) \leq \sum_{i \in I}\left(r+2-d_{i}-l_{i}\right)
$$

Then for each submatrix $A_{i}^{\prime}$ of $A^{\prime}$ where $A_{i}^{\prime}$ is the following :

$$
A_{i}^{\prime}=\left[\begin{array}{ccccc}
A_{y_{i}, l_{y_{i}}} & 0 & \ldots & x_{y_{i}, l_{y_{i}}} & 0 \\
\vdots & \vdots & \vdots & \vdots & \\
A_{y_{i}, r+1-d_{y_{i}}} & 0 & \ldots & x_{y_{i}, r+1-d_{y_{i}}} & 0
\end{array}\right]
$$

Since $r+1-\sum_{i \in I}\left(r+1-d_{i}-\ell_{i}\right) \leq d_{I}$, there exists $\left\{0 \leq t_{i} \leq r+1-d_{i}-\ell_{i}\right\}_{i \in I}$ with $\sum_{i=1}^{|I|} t_{i}=\left[\sum_{i \in I}(r+\right.$ $\left.\left.1-d_{i}-\ell_{i}\right)\right]-\left(r+1-d_{I}\right) \geq 0$,

$$
A_{I, 2}=\left[\begin{array}{ccccc}
A_{y_{1}, \ell_{y_{1}}} & x_{y_{1}, \ell_{y_{1}}} & 0 & & \\
\vdots & \vdots & \vdots & 0 & \\
A_{y_{1}, r+1-d_{y_{1}}-t_{y_{1}}} & x_{y_{1}, r+1-d_{y_{1}}-t_{y_{1}}} & 0 & & 0 \\
A_{y_{k}, l_{y_{k}}} & 0 & \ldots & x_{y_{k}, l_{y_{k}}} & 0 \\
\vdots & \vdots & \vdots & \vdots & 0 \\
A_{y_{k}, r+1-d_{y_{k}}-t_{y_{k}}} & 0 & \ldots & x_{y_{k}, r+1-d_{y_{k}}-t_{y_{k}}} & x_{y_{|I|}, \ell_{|I|}} \\
A_{y_{|I|}, l_{|| I \mid}} & 0 & \ldots & 0 & \\
\vdots & \vdots & \vdots & 0 & x_{y_{|I|}, r+1-d_{y_{|I|}}-t_{|| I \mid}}
\end{array}\right]
$$

whose number of rows is $\left[\sum_{i \in I}\left(r+2-d_{i}-\ell_{i}\right)\right]-\left[\left(\sum_{i \in I}\left(r+1-d_{i}-\ell_{i}\right)\right)-\left(r+1-d_{I}\right)\right]=r+1-d_{I}+|I|$. 
Consider the following matrix:

$$
A_{I, 3}=\left[\begin{array}{c}
A_{y_{1}, \ell_{y_{1}}+1} \\
\vdots \\
A_{y_{1}, r+1-d_{y_{1}}-t_{y_{1}}} \\
A_{y_{k}, l_{y_{k}}+1} \\
\vdots \\
A_{y_{k}, r+1-d_{y_{k}}-t_{y_{k}}} \\
A_{y_{|I|}, l_{y_{|I|}}+1} \\
\vdots \\
A_{y_{|I|}, r+1-d_{y_{|I|}}-t_{y_{|I|}}}
\end{array}\right]
$$

One can see that $A_{I, 3}$ has $r+1-d_{I}$ rows and also by the irreduncy condition and genericity setup, $\operatorname{Rank} A_{I, 3}=r+1-d_{I}$. Also note that $A_{I, 3}$ has $(r+1)$ columns so there exists a $\left(r+1-d_{I}\right) \times(r+1-$ $d_{I}$ ) submatrix of $A_{I, 3}$ with full rank and denote this matrix as $A_{I, 4}$.

Now we take $\left(r+1-d_{I}+|I|\right) \times\left(r+1-d_{I}+|I|\right)$ submatrix of $A^{\prime}$ such that the coefficient of $\Pi_{y_{i} \in I} x_{i, y_{i}}$ (which is a irredundent generator for $I_{p}$ ) is $\operatorname{det} A_{I, 4}$ which is not zero. Thus the lex initial term is $\Pi_{y_{i} \in I} x_{i, y_{i}}$. Thus the lemma follows.

Lemma 4.13. - $\operatorname{in}_{\prec} I_{f}=I_{O}$

Proof: The closed subscheme $X_{\text {inI }_{f}}$ cut by in $\prec I_{f}$ is a flat degeneration of the subscheme $X\left(I_{f}\right)$, thus the multi-degrees of $X\left(\operatorname{in}_{\prec} I_{f}\right)$ and $X\left(I_{f}\right)$ are the same. Also note that $X\left(I_{o}\right)$ and $X\left(I_{f}\right)$ have the same multi-degrees, therefore, $X\left(\operatorname{in}_{\prec} I_{f}\right)$ and $X\left(I_{o}\right)$ have the same multi-degrees.

Since $I_{o} \subset \operatorname{in}_{\prec} I_{f}$, thus $X\left(\operatorname{in}_{\prec} I_{f}\right) \subset X\left(I_{o}\right)$, and $X\left(I_{o}\right)$ is reduced and equidimensional. Therefore $X\left(\operatorname{in}_{\prec} I_{f}\right)=X_{I_{o}}$. Thus the lemma follows.

Corollary 4.14. - The closure of the image of the rational map represents the functors $\mathfrak{F}_{1}$ and $\mathfrak{F}_{2}$. In particular, it is Cohen-Macaulay.

Proof: Recall that the closed subscheme in Description II is cut by $I_{f}$, which represents $\mathfrak{F}_{2}$. Since by Lemma 4.13 we have $\operatorname{in}_{\prec} I_{f}=I_{o}$, which is square free, the variety cut by $I_{f}$ is reduced. The closed points of the scheme of Description II is the same as the closed point of the moduli functor $\mathfrak{F}_{1}$, whose closed points are proven to be the closure of the image of the rational map in Theorem 2.1. The closure of the image of the rational map is also reduced, and therefore is identical with the variety cut by $I_{f}$, which represent the isomorphic moduli functors $\mathfrak{F}_{1}$ and $\mathfrak{F}_{2}$.

In Corollary 3.8, it shows that $\mathfrak{F}_{1}$ is Cohen-Macaulay, then so is the closure of the image of the rational map. 


\section{HILBERT POLYNOMIAL OF $X\left(V_{1}, \cdots, V_{n}\right)$}

It is a natural question to ask after knowing the multi-degrees, what the Hilbert-Polynomial is. In this section, we are going to compute the Hilbert polynomial instead of counting monomials but using degeneration techniques to give a very intuitive argument.

Recall the Hilbert polynomial of product of projective spaces $\Pi_{i=1}^{n} \mathbb{P}^{\ell_{i}}$ is equal to $\Pi_{i=1}^{n}\left(\begin{array}{c}u_{i}+\ell_{i} \\ \ell_{i}\end{array}\right)$ where $u_{i}$ 's are variables.

And for $A$ and $B$ two closed reduced subscheme of the same product of projective spaces, let $H P(\mathscr{X})$ be the Hilbert polynomial of $\mathscr{X}$, a reduced subscheme of a product of projective spaces, then we have $H P(A \cup B)=H P(A)+H P(B)-H P(A \cap B)$. What about for $H P(A \cup B \cup C)$ ? Plugging in the same formula, we have $H P(A \cup B \cup C)=H P(A \cup B)+H P(C)-H P((A \cup B) \cap C)$. In general we shouldn't expect that $H P((A \cup B) \cap C)=H P((A \cap C) \cup(B \cap C))$. In our case, each variety is cut out by a monomial ideal whose generators a degree one monomials, for which we have the following easy lemma:

Lemma 5.1. - Let $I_{1}, \cdots, I_{n}$ be degree one monomial ideals of a multi-variable polynomial ring. Then

$$
\left(I_{1} \cap I_{2} \cap \cdots \cap I_{n-1}\right)+I_{n}=\left(I_{1}+I_{n}\right) \cap I_{2} \cdots \cap I_{n-1}+I_{n}
$$

Proof: Let $J$ be a monomial ideal of the same polynomial ring, then I claim $\left(I_{1}+I_{n}\right) \cap J=\left(I_{1} \cap J\right)+$ $\left(I_{n} \cap J\right)$. Let $M_{J}$ be a minimal set of monomial generators of $J$. And let $S_{I_{1}}$ and $S_{I_{n}}$ be the degree one monomial generators of $I_{1}$ and $I_{n}$ respectively.

Generally for a degree one monomial ideal $I^{1}$ and its degree one generating set $S_{I^{1}}$, let $S_{I^{1}} \star M_{J}$ be the following set of monomials:

$$
\left\{a b: a \in S_{I^{1}}, b \in M_{J} \text { with } a \nmid b\right\} \bigcup\left\{b \mid \exists a \in S_{I^{1}} \text { with } a \mid b\right\}
$$

Therefore, it is not hard to see that $S_{I^{1}} \star M_{J}$ is a generating set of $I^{1} \cap J$, and also if $S_{I^{1}}=A \cup B$, then $S_{I^{1}} \star M_{J}=A \star M_{J} \cup B \star M_{J}$.

Since $\left(I_{1}+I_{n}\right)$ is also a degree one monomial ideal, generating set of $\left(I_{1}+I_{n}\right) \cap J$ can be written as $S_{I_{1}+I_{n}} \star M_{J}$. By the same token, a generating set for $\left(I_{1} \cap J\right)+\left(I_{n} \cap J\right)$ is $\left(S_{I_{1}} \star M_{J}\right) \cup\left(S_{I_{n}} \star M_{J}\right)$. Also notice that $S_{I_{1}+I_{n}}=S_{I_{1}} \cup S_{I_{2}}$, thus we have

$$
\begin{aligned}
S_{I_{1}+I_{n}} \star M_{J} & =\left(S_{I_{1}} \cup S_{I_{2}}\right) \star M_{J} \\
& =\left(S_{I_{1}} \star M_{J}\right) \cup\left(S_{I_{n}} \star M_{J}\right)
\end{aligned}
$$

So a generating set $S_{I_{1}+I_{n}} \star M_{J}$ of $\left(I_{1}+I_{n}\right) \cap J$ is the same as a generating set $\left(S_{I_{1}} \star M_{J}\right) \cup\left(S_{I_{n}} \star M_{J}\right)$ of $\left(I_{1} \cap J\right)+\left(I_{n} \cap J\right)$, then the claim follows. 
Now let's go back to the statement of the lemma, note that $I_{2} \cap \cdots \cap I_{n-1}$ is also a monomial ideal, then by the claim we just proved, we have

$$
\left(I_{1}+I_{n}\right) \cap I_{2} \cdots \cap I_{n-1}=\left(I_{1} \cap I_{2} \cap \cdots \cap I_{n-1}\right)+I_{n} \cap I_{2} \cdots \cap I_{n-1},
$$

but also $I_{n} \cap I_{2} \cdots \cap I_{n-1} \subset I_{n}$, finally we have

$$
\begin{aligned}
\left(I_{1}+I_{n}\right) \cap I_{2} \cdots \cap I_{n-1}+I_{n} & =\left(I_{1} \cap I_{2} \cap \cdots \cap I_{n-1}\right)+I_{n} \cap I_{2} \cdots \cap I_{n-1}+I_{n} \\
& =\left(I_{1} \cap I_{2} \cap \cdots \cap I_{n-1}\right)+I_{n}
\end{aligned}
$$

Then the lemma follows.

Corollary 5.2. - Let $I_{1}, \cdots, I_{n}$ be degree one monomial ideals of a multi-variable polynomial ring. Then

$$
\left(I_{1} \cap I_{2} \cap \cdots \cap I_{n-1}\right)+I_{n}=\left(I_{1}+I_{n}\right) \cap\left(I_{2}+I_{n}\right) \cdots \cap\left(I_{n-1}+I_{n}\right)
$$

Proof: By Lemma5.1 $\left(I_{1} \cap I_{2} \cap \cdots \cap I_{n-1}\right)+I_{n}=\left(I_{1}+I_{n}\right) \cap I_{2} \cdots \cap I_{n-1}+I_{n}$, note that $\left(I_{1}+I_{n}\right)$ is also a degree one monomial ideal, and $\left(I_{1}+I_{n}\right) \cap I_{2} \cdots \cap I_{n-1}+I_{n}=I_{2} \cap\left(I_{1}+I_{n}\right) \cdots \cap I_{n-1}+I_{n}$, applying Lemma 5.1, we have

$$
\begin{aligned}
\left(I_{1} \cap I_{2} \cap \cdots \cap I_{n-1}\right)+I_{n} & =\left(I_{1}+I_{n}\right) \cap I_{2} \cdots \cap I_{n-1}+I_{n} \\
& =I_{2} \cap\left(I_{1}+I_{n}\right) \cdots \cap I_{n-1}+I_{n} \\
& =\left(I_{2}+I_{n}\right) \cap\left(I_{1}+I_{n}\right) \cdots \cap I_{n-1}+I_{n} \\
& =\left(I_{1}+I_{n}\right) \cap\left(I_{2}+I_{n}\right) \cdots \cap I_{n-1}+I_{n}
\end{aligned}
$$

Then by the same token and repeated use of Lemma 5.1, we finally have

$$
\left(I_{1} \cap I_{2} \cap \cdots \cap I_{n-1}\right)+I_{n}=\left(\left(I_{1}+I_{n}\right) \cap\left(I_{2}+I_{n}\right) \cap \cdots \cap\left(I_{n-1}+I_{n}\right)\right)+I_{n} .
$$

Also let's note that $I_{n} \subset\left(\left(I_{1}+I_{n}\right) \cap\left(I_{2}+I_{n}\right) \cap \cdots \cap\left(I_{n-1}+I_{n}\right)\right)$. Thus we obtain:

$$
\begin{aligned}
\left(I_{1} \cap I_{2} \cap \cdots \cap I_{n-1}\right)+I_{n} & =\left(\left(I_{1}+I_{n}\right) \cap\left(I_{2}+I_{n}\right) \cap \cdots \cap\left(I_{n-1}+I_{n}\right)\right)+I_{n} \\
& =\left(I_{1}+I_{n}\right) \cap\left(I_{2}+I_{n}\right) \cdots \cap\left(I_{n-1}+I_{n}\right)
\end{aligned}
$$

Finally we can get the following convenient property for us to compute the Hilbert polynomial.

Proposition 5.3. - Let $P_{i}, 1 \leq i \leq n$ be closed subschemes of a product of projective spaces cut by degree one monomial ideals, then

$$
H P\left(\left(P_{1} \cup P_{2} \cup \cdots \cup P_{n-1}\right) \bigcap P_{n}\right)=H P\left(\left(P_{1} \cap P_{n}\right) \cup\left(P_{2} \cap P_{n}\right) \cup \cdots \cap\left(P_{n-1} \cap P_{n}\right)\right)
$$

Proof: It is a direct application of Corollary 5.2.

Theorem 5.4. - The multi-variable Hilbert polynomial of the variety cut by $I_{f}$ equals

$$
\sum_{S \subset M(p)}(-1)^{|S|-1} \Pi_{i=1}^{n}\left(\begin{array}{c}
u_{i}+\ell_{S, i} \\
\ell_{S, i}
\end{array}\right)
$$

where $\ell_{S, i}$ is the smallest $i$-th component of all elements of $S$. 
Proof: Hilbert Polynomial is preserved under flat degeneration, so it suffices to compute the Hilbert polynomial of in $_{\prec} I_{f}$, whose prime decomposition has already been computed in Section 4 , i.e.,

$$
\operatorname{in}_{\prec} I_{f}=\bigcap_{\left(m_{1}, \cdots, m_{n}\right) \in M(p)} P_{\left(m_{1}, \cdots, m_{n}\right)}
$$

For $\left(m_{1}, \cdots, m_{n}\right) \in M\left(r^{\prime}\right)$, we have

$$
P_{\left(m_{1}, \cdots, m_{n}\right)}=<x_{1,1}, x_{1,2}, \cdots, x_{1, r-d_{1}-m_{1}}, \ldots, x_{i, 1}, \cdots, x_{i, r-d_{i}-m_{i}}, \ldots, x_{n, 1}, \cdots, x_{n, r-d_{n}-m_{n}}>.
$$

Thus the variety cut by in $_{\prec} I_{f}$ is a union of $\Pi_{i=1}^{n} \mathbb{P}^{m_{i}}$ with $\left(m_{1}, \cdots, m_{n}\right) \in M\left(r^{\prime}\right)$. By a direct computation, for $S \subset M(p)$

$$
\bigcap_{\left(m_{1}, \cdots, m_{n}\right) \in S} \Pi_{i=1}^{n} \mathbb{P}^{m_{i}}=\Pi_{i=1}^{n} \mathbb{P}^{l} s_{i}
$$

where $\ell_{S, i}$ is the smallest integer among the $i$-th components of integer vectors in $S$. Then this theorem will follow from the following claim:

Given $T_{1}, \cdots, T_{n}$ subvarieties of $\Pi_{i=1}^{n} \mathbb{P}\left(V / V_{i}\right)$, where $T_{i}$ 's are cut by degree one monomial ideal, then

$$
H P\left(\cup_{i=1}^{n} T_{i}\right)=\sum_{I \subset[n]}(-1)^{|I|-1} H P\left(\cap_{i \in I} T_{i}\right)
$$

When $n=2$, we can deduce this from $H P(A \cup B)=H P(A)+H P(B)-H P(A \cap B)$ for any closed subvariety $A$ and $B$.

When $n=k$, assume the claim is true. For $n=k+1$, we have

$$
H P\left(\cup_{i=1}^{k+1} T_{i}\right)=H P\left(T_{1}\right)+H P\left(\cup_{i=2}^{k+1} T_{i}\right)-H P\left(P_{1} \cap\left(\cup_{i=2}^{k+1} T_{i}\right)\right)
$$

By Proposition 5.3

$$
H P\left(T_{1} \cap\left(\cup_{i=2}^{k+1} T_{i}\right)\right)=H P\left(\cup_{i=2}^{k+1}\left(T_{1} \cap T_{i}\right)\right) .
$$

Since $T_{1} \cap T_{i}$ is also cut by degree one monomial ideal, we can use induction hypothesis to get:

$$
H P\left(\cup_{i=2}^{k+1}\left(T_{1} \cap T_{i}\right)\right)=\sum_{I^{\prime}}(-1)^{\left(\left|I^{\prime}\right|-1\right)} H P\left(\cap_{I^{\prime}}\left(T_{1} \cap T_{i}\right)\right)=\sum_{I^{\prime}}(-1)^{\left(\left|I^{\prime}\right|-1\right)} H P\left(\cap_{i \in I^{\prime}}\left(T_{i}\right) \cap T_{1}\right),
$$

where $I^{\prime}$ is a subset of $[k+1] \backslash\{1\}$. Therefore, we finally have

$$
\begin{aligned}
H P\left(\cup_{i=1}^{k+1} P_{i}\right) & =H P\left(T_{1}\right)+H P\left(\cup_{i=2}^{k+1} T_{i}\right)-H P\left(P_{1} \cap\left(\cup_{i=2}^{k+1} T_{i}\right)\right) \\
& =H P\left(T_{1}\right)+H P\left(\cup_{i=2}^{k+1} T_{i}\right)-\sum_{I^{\prime} \subset[k+1] \backslash\{1\}}(-1)^{\left(\left|I^{\prime}\right|-1\right)} H P\left(\cap_{i \in I^{\prime}}\left(T_{i}\right) \cap T_{1}\right) \\
& =H P\left(T_{1}\right)+\sum_{I^{\prime} \subset[k+1] \backslash\{1\}}(-1)^{\left(\left|I^{\prime}\right|-1\right)} H P\left(\cap_{i \in I^{\prime}} T_{i}\right)+\sum_{I^{\prime} \subset[k+1] \backslash\{1\}}(-1)^{\left(\left|I^{\prime}\right|+1\right)-1} H P\left(\cap_{i \in I^{\prime}}\left(T_{i}\right) \cap T_{1}\right) \\
& =\sum_{I \subset[k+1]}(-1)^{(|I|-1)} H P\left(\cap_{i \in I} T_{i}\right),
\end{aligned}
$$

thus the claim follows. 


\section{References}

[1] Michel Brion. Multiplicity-free subvarieties of flag varieties. Contemporary Math, 331:13-23.

[2] David Eisenbud. Commutative Algebra: with a View Toward Algebraic Geometry. Springer.

[3] Jason Morton. Relations among conditional probabilities. To appear in J. Symbolic Computation.

[4] G. Mustafin. Non-archimedean uniformization. n. Math. USSR Sbornik, 34:187-214, 1978.

[5] Eduardo Esteves; Brian Osserman. Abel maps and limit linear series. Rendiconti del Circolo Matematico di Palermo, 62:79-95, 2013.

[6] Chris Aholt; Bernd Sturmfels; Rekha Thomas. A Hilbert scheme in computer vision. to appear in Canadian Journal of Mathematics.

[7] Dustin Cartwright; Mathias Häbich; Bernd Sturmfels; Annette Werner. Mustafin varieties. Sel. Math. New Ser., 1:757-793, 2011.

\section{Binglin $\mathrm{Li}$}

Department of Mathematics

University of California, Davis

One Shields Avenue, Davis California 95616

blnli@math.ucdavis.edu 